Artículo científico

Volumen 33(1): Artículo 44508, 2022

e-ISSN 2215-3608, doi:10.15517/am.v33i1.44508

https://revistas.ucr.ac.cr/index.php/agromeso/index

\title{
Rendimiento e inclusión de ensilaje de avena forrajera (Avena sativa L.) AV25 en dietas para ganado lechero (Bos primigenius Taurus) ${ }^{1}$
}

\section{Performance and silage inclusion of oat forage (Avena sativa L.) AV25 in diets for dairy cattle (Bos primigenius Taurus)}

\author{
Luis Fernando Campuzano-Duque ${ }^{2}$,Edwin Castro-Rincón ${ }^{3}$, Javier Castillo-Sierra ${ }^{4}$, Daniel Torres-Cuesta ${ }^{4}$, \\ David Nieto-Sierra ${ }^{3}$, Paola Portillo-López ${ }^{3}$
}

1 Recepción: 11 de diciembre, 2020. Aceptación: 1 de julio, 2021. Este trabajo formó parte de un proyecto asociado al Programa de Mejoramiento de Forrajes en Colombia y financiado por el Ministerio de Agricultura y Desarrollo Rural (MADR) y la Corporación Colombiana de Investigación Agropecuaria (AGROSAVIA). Colombia.

2 Corporación Colombiana de Investigación Agropecuaria (AGROSAVIA), Centro de Investigación La Libertad, Villavicencio, Colombia. lcampuzano@agrosavia.co (autor para correspondencia; https://orcid.org/0000-0003-1146-4591).

3 Corporación Colombiana de Investigación Agropecuaria (AGROSAVIA), Centro de Investigación Obonuco, Pasto, Colombia. ecastro@ agrosavia.co (https://orcid.org/0000-0001-9841-8242); dnieto@agrosavia.co (https://orcid.org/0000-0003-3033-3079); pportillo@agrosavia. co (https://orcid.org/0000-0003-1189-9173).

4 Corporación Colombiana de Investigación Agropecuaria (AGROSAVIA), Centro de Investigación Tibaitatá, Bogotá, Colombia. jcastillo@ agrosavia.co (https://orcid.org/0000-0003-0797-3908); dtorres@agrosavia.co (https://orcid.org/0000-0001-9101-0543).

\section{Resumen}

Introducción. Utilizar ensilaje de avena forrajera (Avena sativa L.) en la ganadería de leche en épocas deficitarias de alimento, permite mitigar pérdidas de biomasa por sequía en el trópico alto colombiano. Objetivos. 1) Determinar el rendimiento en materia seca (RMS) de cinco genotipos de avena forrajera y la estabilidad fenotípica y la calidad bromatológica de su ensilaje, y 2) evaluar la inclusión de ensilaje del genotipo AV25-T en dietas para ganado lechero. Materiales y métodos. Se realizaron en el trópico alto colombiano dos experimentos: 1) prueba de evaluación agronómica (octubre de 2016 a febrero de 2017) en un diseño de bloques completos al azar con cinco genotipos, ocho localidades y cuatro repeticiones; se determinó el rendimiento en materia seca (RMS) y en ensilaje los contenidos de proteína, fibra en detergente neutro (FDN) y nutrientes totales digestibles (NTD) y 2) estudio de respuesta animal (enero 2018) con la inclusión del genotipo AV25-T en dietas, en un diseño Crossover simple con dos factores: animal (5) y dietas (3); las dietas fueron: D1: solo pradera; D2: $35 \%$ de ensilaje AV25-T + pastoreo y D3: $65 \%$ de ensilaje AV25-T + pastoreo. Se determinó en la leche la producción, la proteína, la grasa y los sólidos totales, y en el animal el cambio de peso. Resultados. AV25-T presentó adaptación con el mayor RMS. La inclusión de ensilaje de AV25-T en la dieta animal, mostró un cambio significativo en el peso del animal (0,1 y 0,25 kg con D2 y D3), el rendimiento de leche (27,2 - 29,7 L/vaca/día con D3 y D2) y el contenido de grasa (3,2 - 3,9 \% con D3 y D2), sin detrimento en proteína y sólidos totales. Conclusión. El uso del ensilaje de avena forrajera AV25-T permitió en vacas lecheras Holstein incrementos en el peso animal, el rendimiento de leche y el contenido grasa sin afectar la proteína y los sólidos totales.

Palabras clave: composición de la leche, cultivos forrajeros, cultivos de cereales, cereal forrajero, experimentos de alimentación, zonas tropicales. 


\begin{abstract}
Introduction. The use of forage oat silage (Avena sativa L.) in dairy cattle in times of feed deficit, allows mitigating biomass losses due to drought in the Colombian high tropics. Objectives. 1) To determine the dry matter yield (DMY) of five forage oat genotypes and the phenotypic stability and the bromatological quality of their silage, and 2) to evaluate the inclusion of the AV25-T genotype silage in diets for dairy cattle. Materials and methods. In the Colombian high tropics two experiments were performed: 1) agronomic evaluation test (October 2017 and February 2018) in a randomized complete block design with five genotypes, eight locations, and four replicates; dry matter yield (DMY) and protein, neutral detergent fiber (NDF) and total digestible nutrients (TDN) contents in silage were determined, and 2) animal response study (January 2018) with the inclusion of the AV25-T genotype in diets, in a simple crossover design with two factors: animal (5) and diets (3); the diets were: D1: only meadow; D2: $35 \%$ AV25-T silage + grazing, and D3: $65 \%$ AV25-T silage + grazing. Milk yield, protein, fat, total solids, were determined in the milk, and weight change was determined in the animals. Results. AV25-T presented adaptation with the highest DMY. The inclusion of AV25-T silage in the animal diet showed a significant change in animal weight $(0.1$ and $0.25 \mathrm{~kg}$ with D2 and D3), milk yield (27.2 - 29.7 L/cow/day with D3 and D2), and the fat content (3.2 - $3.9 \%$ with D3 and D2), without detriment in protein and total solids. Conclusion. The use of AV25-T forage oats in silage allowed increases in animal weight, milk yield, and fat content in Holstein dairy cows without affecting protein and total solids in milk.
\end{abstract}

Keywords: milk composition, feed crops, cereal crops, feed cereal, feeding experiments, tropical zones.

\title{
Introducción
}

En Colombia, 400000 ganaderos tienen una producción diaria de 15 millones de litros de leche (Departamento de Administración Nacional de Estadística, 2015) con una concentración del 42,5 \% en los altiplanos Cundiboyacense, Nariño y Antioquia, con predominio de pequeños predios de economía campesina (Carulla \& Ortega, 2016). No obstante, la sequía y las heladas asociadas con la variabilidad climática, han afectado la productividad, con pérdidas económicas de 80,2 millones de litros de leche por año, en 150 mil hectáreas (Arteaga \& Burbano, 2017; CardonaIglesias et al., 2019).

La producción de leche en el trópico alto está basada en pasturas naturalizadas con el pasto kikuyo (Cenchrus clandestinus H.) (Arteaga \& Burbano, 2017; Castro-Rincón et al., 2020; Vargas-Martínez et al., 2018) y como estrategia complementaria se usa la avena, como forraje verde en pastoreo, heno o ensilaje (Kapoor \& Singh, 2020; Tulu et al., 2020); es de rápido crecimiento, con un potencial de rendimiento de forraje verde de 60 a $80 \mathrm{t} \mathrm{ha}^{-1}$ (Wada et al., 2019). La avena, además de su potencial de rendimiento, calidad bromatológica y palatabilidad, presenta tolerancia al frío, resistencia a la sequía y adaptabilidad (Sharma et al., 2016).

Los efectos de las dietas de suplementación animal con ensilaje de avena forraje en sustitución del pasto kikuyo, sobre la producción de leche, el peso animal y la calidad nutricional (proteína y grasa), son variables, en algunos casos positiva y en otros negativa por la compleja interacción del tipo de biomasa, su proporción y tiempo de consumo, así como la razade la vaca y el tercio de la lactancia en que se encuentre (Mojica et al., 2009).

En el trópico alto colombiano, para la mitigación de la sequía y las heladas en el componente animal, es común recomendar el uso del ensilaje de avena (Avena sativa L.) para compensar la baja oferta de biomasa del pasto kikuyo en época de sequía en la subregión Altiplano Cundiboyacense (SRC) y subregión Altiplano de Nariño (SRN); esta recomendación está dada en tres variedades forrajeras comerciales: Avenar, Cajicá y Cayuse. No obstante, estas variedades han demostrado en los últimos años susceptibilidad al volcamiento, a la roya de la hoja (Puccinia coronataf. sp avenae) y roya del tallo (Puccinia gramini f. sp. avenae) (Campuzano et al., 2020). En la 
actualidad, el programa de mejoramiento de forrajes de la Corporación Colombiana de Investigación Agropecuaria (AGROSAVIA), posee dos genotipos élites (AV25-S y AV25-T) que demostraron en ensayos previos en el trópico alto colombiano, en los altiplanos Cundiboyacense y de Nariño, un desempeño superior en rendimiento de forraje en relación con Avenar, Cajicá y Cayuse, con atributos adicionales de resistencia al volcamiento, a la roya de la hoja y del tallo y precocidad (Campuzano et al., 2020).

Para complementar la información de los dos genotipos élite de avena en términos de calidad bromatológica en condiciones de ensilaje y su respuesta animal al consumo, AGROSAVIA desarrolló esta investigación con los objetivos de: 1) determinar el rendimiento en materia seca de cinco genotipos de avena forrajera y la estabilidad fenotípica y la calidad bromatológica de su ensilaje, y 2) evaluar la inclusión de ensilaje del genotipo AV25-T en dietas para ganado lechero.

\section{Materiales y métodos}

Entre octubre 2016 y febrero 2017, se realizó una prueba de evaluación agronómica (PEA) ajustada a la resolución 067516 (Instituto Colombiano Agropecuario, 2020, en un diseño de bloques completos al azar con dos factores: a) genotipos (5) y localidades (8) y cuatro repeticiones. Las localidades correspondieron cuatro a la subregión Altiplano Cundiboyacense (SRC) y cuatro a la subregión Altiplano de Nariño (SRN); con la siguiente

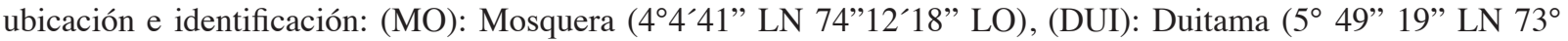

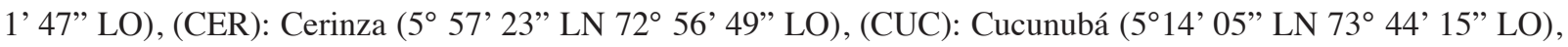

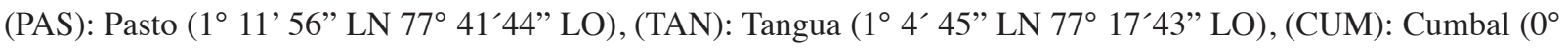

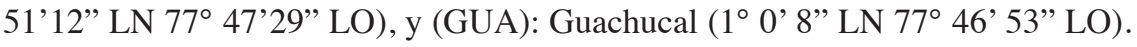

Las localidades en la SRC se ubicaron en altitudes entre 2543 y $2760 \mathrm{~m}$, con una temperatura media anual entre 16,9 y $17,4^{\circ} \mathrm{C}$, mientras que en la SRN se localizaron entre 2710 y $3184 \mathrm{msnm}$, con una temperatura media anual entre 12,6 a $14,5^{\circ} \mathrm{C}$. Con base en estas dos variables de clima y según la clasificación Caldas-Lang (Gutiérrez, 1991), todas las localidades en ambas subregiones se clasificaron como Fsh (clima frío semihúmedo) con excepción de CUM que se clasificó como Mfh (clima muy frío húmedo). Las localidades presentaron, desde la siembra hasta la cosecha de forraje verde, una precipitación en mm de 618,0 en Mosquera, 300,0 en Duitama, 386,8 en Cerinza, 526,8 en Cucunubá, 380,6 en Pasto, 379,7 en Cumbal, 210,5 en Tangua y 598,7 en Guachucal (Cuadro 1).

Para conocer la fertilidad del suelo (Cuadro 2) y realizar los correctivos y la fertilización, en cada localidad se tomaron muestras de suelo a una profundidad de 0 a $20 \mathrm{~cm}$, que permitió determinar las características físicas: textura (método del hidrómetro o Bouyoucos) y químicas: $\mathrm{pH}$ (método potenciómetro), materia orgánica (\%) (método de Walkley y Black), fósforo $\left(\mathrm{mg} \mathrm{kg}^{-1}\right)$ (método Bray II), calcio $\left(\mathrm{cmol} \mathrm{kg}{ }^{-1}\right)$, magnesio $\left(\mathrm{cmol} \mathrm{kg}^{-1}\right) \mathrm{y}$ potasio $\left(\mathrm{cmol} \mathrm{kg}^{-1}\right)$ (extracción con acetato de amonio pH 7 y cuantificación por absorción atómica). Estos análisis se realizaron en el laboratorio de química de suelos, aguas y plantas de AGROSAVIA con la Norma Técnica de Calidad NTC-2005.

En el acondicionamiento del suelo, en relación con el $\mathrm{pH}$, en cada localidad, se aplicaron $4 \mathrm{t} \mathrm{ha-1}$ de cal dolomítica y la preparación del suelo se realizó con un pase de arado de cincel vibratorio, dos pases de rastra y un pase de rastra pulidor. La siembra se realizó de forma manual al voleo asociada con una fertilización edáfica basada en el resultado del análisis del suelo y el requerimiento nutricional de la avena forrajera para producir $80 \mathrm{t}$ $\mathrm{ha}^{-1}$. Los productos aplicados fueron compuestos simples en $\mathrm{kg} \mathrm{ha}^{-1}$ de $\mathrm{N}: 150, \mathrm{P}_{2} \mathrm{O}_{5}: 120 ; \mathrm{K}_{2} \mathrm{O}: 90$, Ca: 70, Mg: 25 y Borozinco: 20. El nitrógeno se aplicó fraccionado, a la siembra y al macollamiento (75:75). Se realizó control de malezas, de gramíneas y de hoja ancha, desde el momento de la siembra con una aplicación de preemergente (ácido-4-amino-3,5,6-tricloropicolínico $200 \mathrm{cc} \mathrm{ha}^{-1}$ ) y posemergente (Metsulfuron Metil $15 \mathrm{~g} \mathrm{ha}^{-1}$ ). 
Cuadro 1. Altitud, precipitación y temperatura media ocurrida entre la emergencia a cosecha de forraje de avena (Avena sativa L.), en ocho localidades de la zona Andina colombiana. Corporación Colombiana de Investigación Agropecuaria (AGROSAVIA). $2016-2017$.

Table 1. Altitude, precipitation, and average temperature between the emergence of oat (Avena sativa L.) forage harvest, in eight localities of the Colombian Andean zone. Corporacion Colombiana de Investigacion Agropecuaria (AGROSAVIA). 2016-2017.

\begin{tabular}{|c|c|c|c|c|c|c|c|c|}
\hline \multirow[t]{2}{*}{ Localidad/subregión } & \multicolumn{4}{|c|}{ Cundiboyacense } & \multicolumn{4}{|c|}{ Nariño } \\
\hline & MOS & DUI & CER & CUC & PAS & TAN & CUM & GUA \\
\hline Altitud (msnm) & 2543 & 2760 & 2854 & 2550 & 2710 & 2999 & 3184 & 3180 \\
\hline Temperatura $\left({ }^{\circ} \mathbf{C}\right)$ & 17,4 & 17,2 & 17,1 & 16,9 & 14,5 & 15,2 & 12,6 & 12,4 \\
\hline Precipitación (mm) & 618,0 & 300,0 & 386,8 & 526,8 & 380,6 & 210,5 & 379,7 & 398,7 \\
\hline Agua $^{1}$ & 100,0 & 75,0 & 97,0 & 100,0 & 95,0 & 53,0 & 95,0 & 99,0 \\
\hline
\end{tabular}

Fuente: Instituto de Hidrología, Meteorología y Estudios Ambientales (2017). 1: porcentaje de agua satisfecha sobre la base de requerimiento de $400 \mathrm{~mm}$ (Hussain et al., 2010; Terán-Chaves et al., 2014); localidades: MOS: Mosquera; DUI: Duitama; CER: Cerinza; CUC: Cucunubá; PAS: Pasto; TAN: Tangua; CUM: Cumbal; GUA: Guachucal / Source: Institute of Hydrology, Meteorology, and Environmental Studies (2017). 1: percentage of water satisfied based on a requirement of $400 \mathrm{~mm}$ (Hussain et al., 2010; TeránChaves et al., 2014); localities: MOS: Mosquera; DU: Duitama; CER: Cerinza; CUC: Cucunubá; PAS: Pasto; TAN: Tangua; CUM: Cumbal; GUA: Guachucal.

Cuadro 2. Características fisicoquímicas del suelo en ocho localidades de la zona Andina. Corporación Colombiana de Investigación Agropecuaria (AGROSAVIA). Altiplano Cundiboyacense y de Nariño. 2016-2017.

Table 2. Physicochemical characteristics of the soil in eight localities of the Andean zone. Corporacion Colombiana de Investigacion Agropecuaria (AGROSAVIA). Altiplano Cundiboyacense and Nariño. 2016-2017.

\begin{tabular}{|c|c|c|c|c|c|c|c|}
\hline \multirow[b]{2}{*}{ Localidad } & \multirow[b]{2}{*}{ Textura } & \multirow[b]{2}{*}{$\mathbf{p H}$} & \multirow{2}{*}{$\begin{array}{c}\text { Materia } \\
\text { orgánica } \%\end{array}$} & \multirow{2}{*}{$\begin{array}{l}\text { Fósforo } \\
\text { mg kg }^{-1}\end{array}$} & Calcio & Magnesio & Potasio \\
\hline & & & & & \multicolumn{3}{|c|}{$\mathrm{Cmol} \mathrm{kg}^{-1}$} \\
\hline Mosquera & FAr & 5,7 & $5,1 \mathrm{M}$ & $24,7 \mathrm{~A}$ & $5,2 \mathrm{~A}$ & $3,6 \mathrm{~A}$ & $0,4 \mathrm{~A}$ \\
\hline Duitama & FArA & 5,7 & 7,7 M & $23,5 \mathrm{~A}$ & $4,9 \mathrm{~A}$ & $3,6 \mathrm{~A}$ & $0,9 \mathrm{~A}$ \\
\hline Cerinza & FArA & 5,7 & 7,0 M & $20,9 \mathrm{~A}$ & $4,3 \mathrm{~A}$ & $0,3 \mathrm{~B}$ & $0,1 \mathrm{~B}$ \\
\hline Cucunubá & FAr & 5,7 & $5,7 \mathrm{M}$ & $38,9 \mathrm{~A}$ & $6,4 \mathrm{~A}$ & $1,1 \mathrm{~B}$ & $0,6 \mathrm{~A}$ \\
\hline Pasto & FAr & 5,9 & $8,9 \mathrm{~A}$ & $17,5 \mathrm{~A}$ & $8,8 \mathrm{~A}$ & $1,8 \mathrm{~m}$ & $0,2 \mathrm{~B}$ \\
\hline Tangua & FAr & 5,2 & $11,0 \mathrm{~A}$ & $17,1 \mathrm{~A}$ & $4,0 \mathrm{M}$ & $0,6 \mathrm{~B}$ & $0,7 \mathrm{~A}$ \\
\hline Cumbal & FAr & 5,2 & $16,0 \mathrm{~A}$ & $18,3 \mathrm{~A}$ & 7,4 A & $1,6 \mathrm{M}$ & $0,7 \mathrm{~A}$ \\
\hline Guachucal & FAr & 5,4 & $8,7 \mathrm{~A}$ & $25,2 \mathrm{~A}$ & $3,4 \mathrm{M}$ & $1,4 \mathrm{M}$ & $0,6 \mathrm{~A}$ \\
\hline
\end{tabular}

FAr: franco arcilloso; FArA: franco arcilloso arenoso; A: alto; M: medio; B: bajo (Riascos, 1998) / FAr : clay loam; FArA: sandy clay loam; A: high; M: medium; B: low (Guerrero-Riascos, 1998).

Los genotipos de avena forrajera (Avena sativa L.) correspondieron a dos materiales élite del programa de mejoramiento genético de forrajes de AGROSAVIA y denominados AV25-S y AV25-T y se incluyeron como testigos tres genotipos que son variedades de uso comercial: Avenar, Cajicá y Cayuse. La unidad experimental estuvo constituida por parcelas de $30 \mathrm{~m}^{2}$ con una densidad de semilla de $80 \mathrm{~kg} \mathrm{ha}^{-1}$. En el estado de grano lechoso (Zadoks, 1974), en un área de $15 \mathrm{~m}^{2}$ en cada tratamiento, localidad y repetición, se realizó la cosecha de forraje para determinar el rendimiento de forraje verde (RFV).

En cada genotipo, localidad y repetición, del forraje verde cosechado, se tomaron dos muestras: 1) una de 300 $\mathrm{g}$, la cual se secó en un horno a $65^{\circ} \mathrm{C}$ durante $72 \mathrm{~h}$ hasta que se obtuvo un peso constante. Este valor obtenido 
denominado contenido de materia seca (CMS) (\%) se usó para determinar el rendimiento en materia seca (RMS) en $\mathrm{t} \mathrm{ha}^{-1}$ con base en el rendimiento de forraje verde (RFV x CMS) y 2) una muestra de 2,0 kg, la cual se sometió a fermentación por 60 días mediante la técnica de microsilo (bolsa plástica de color negro de $2 \mathrm{~mm}$ de diámetro y con sellado hermético).

De cada microsilo se tomó una muestra de $200 \mathrm{~g}$ para el análisis bromatológico en el laboratorio de nutrición animal de AGROSAVIA (Mosquera-Colombia). La composición nutricional en base seca se determinó para: proteína (P), fibra detergente en neutro (FDN), y nutrientes totales digestibles (NTD), estas tres variables se expresaron en porcentaje. Las variables P, FDN, NTD, se obtuvieron con el uso de las ecuaciones NIRS ajustadas por AGROSAVIA (Ariza-Nieto et al., 2017) con el equipo NIRS DS 2500 - FOSS Analítica A/S - Dinamarca.

Con los datos de RMS, P, FDN y NTD se verificaron los supuestos teóricos del análisis de varianza: 1) la prueba de Shapiro \& Wilk (1965), para la homogeneidad de varianza, 2) análisis individual de varianza y la combinación de localidades, y 3) comparación de medias con la prueba de Tukey ( $\mathrm{p}=0,01)$. Los procedimientos y análisis estadísticos se realizaron con el programa SAS versión 9.4 (Statistical Analysis Systems Institute, 2016).

El modelo estadístico utilizado fue el siguiente: Yijk $=\mu+\mathrm{Gi}+\mathrm{Lj}+(\mathrm{GL}) \mathrm{ij}+\mathrm{Bk}(\mathrm{j})+$ Eijk; donde: Yijk $=$ variable de estudio promedio del i-ésimo genotipo obtenido en el j-ésimo localidad y k-ésima repetición; $\mu=$ efecto de la media general; $\mathrm{Gi}$ = efecto del i-ésimo genotipo; $\mathrm{Aj}=$ efecto del $\mathrm{j}$-ésimo localidad; $(\mathrm{GL}) \mathrm{ij}=$ efecto de la interacción entre el i-ésimo genotipo y el j-ésimo localidad; $\mathrm{Bk}(\mathrm{j})$ = efecto de la k- ésima repetición en el j-ésimo localidad y Eijk = efecto aleatorio del error experimental asociado al i-ésimo genotipo en el j-ésimo localidad y K-ésima repetición, según el modelo lineal aditivo.

\section{Prueba de respuesta animal}

Para la prueba de respuesta animal, se utilizó el genotipo AV25-T por haber presentado un rendimiento de forraje verde significativamente superior en pruebas de evaluación agronómica en la SRC y SRN, asociado con resistencia al volcamiento y a la roya de la hoja y del tallo (Campuzano et al., 2020).

El forraje verde requerido para la prueba de respuesta animal se obtuvo con el establecimiento de una parcela semicomercial (agosto a noviembre de 2017) en un área de $6000 \mathrm{~m}^{2}$ con una densidad de $80 \mathrm{~kg} \mathrm{ha}^{-1}$; la cosecha de forraje verde (FV) se realizó a los 109 días en el estado grano lechoso (escala 7,9) (Zadoks et al., 1974). El FV cosechado se depositó en un silo horizontal tipo Bunker de 1,5 m de ancho, 1,0 m de alto y $10 \mathrm{~m}$ de longitud, con muros de hormigón; se comparó el FV con tractor, luego se selló y protegió. La conservación del ensilaje se realizó por 60 días (noviembre a diciembre de 2017), tiempo en el cual se obtuvieron condiciones organolépticas adecuadas de color y olor.

La prueba de respuesta animal se realizó durante la época de verano (enero 2018) en el municipio de TibasosaSRC ( $5^{\circ} 44^{\prime} 53^{\prime \prime}$ LN 72 59’56" LO) por un periodo de 21 días. Se utilizó un diseño "complete block crossover" o crossover simple (Amézquita, 1986; Lui, 2016) con dos factores: unidad de vaca y dietas; la unidad de vaca se constituyó de cinco animales de ganado lechero (Bos primigenius taurus) de la raza Holstein en el primer tercio de lactancia y su rotación asignada al azar en cada dieta tuvo una duración de 7 días, momento en el cual las vacas pasaban a otra dieta; al final de los 21 días todas unidades de vaca tuvieron la oportunidad de obtener las tres dietas. Las dietas suministradas por día a los animales fueron: D1: solo pradera; D2: $35 \%$ (12,0 kg) de ensilaje AV25-T + $65 \%$ pastoreo y D3: $65 \%(25,9 \mathrm{~kg})$ de ensilaje AV25-T + $35 \%$ pastoreo. La pradera utilizada en todas las dietas fue el pasto Kikuyo (Cenchrus clandestinus H.) y la suplementación en los tratamientos D2, D3 fue un concentrado comercial $(6 \mathrm{~kg})$, semilla de algodón $(0,5 \mathrm{~kg})$ y harina de alfalfa $(0,5 \mathrm{~kg})$.

$\mathrm{El}$ área de la pradera de kikuyo ofrecida en cada dieta se ajustó cada semana con un aforo de disponibilidad y de residualidad de forraje a la salida de cada unidad de vaca y el consumo diario total en cada dieta se calculó con 
base en el 3,2 \% del peso vivo animal. AV25-T se ofreció a cada unidad de vaca en comederos portátiles, con ración diaria de cada dieta en dos fracciones: $80 \%$ en la mañana y $40 \%$ en la tarde, después de cada ordeño; el ensilaje que no fue consumido por la unidad de vaca fue pesado y registrado para determinar el consumo real.

Al final de cada dieta en cada unidad de vaca se determinaron: producción de leche (PL), durante los siete días de evaluación y expresada en 1 vaca $^{-1}$ día $^{-1}$ y con una muestra de $100 \mathrm{ml}$ de leche se evaluó la calidad composicional de proteína $(\mathrm{P})$, grasa $(\mathrm{G})$ y sólidos totales $(\mathrm{ST})$ expresado en porcentaje y obtenido con el método de espectroscopía infrarrojo (Ariza-Nieto et al., 2017). Se determinó el peso inicial (día 1) y final (día 7) con el método de la cinta para calcular el cambio en peso animal (CPA) expresado en $\mathrm{kg} \mathrm{vaca}^{-1}$ día $^{-1}$.

El modelo estadístico utilizado fue: $\mathrm{Y}_{\mathrm{ij}}=\mathrm{U}+\mathrm{Si}+\mathrm{T}_{\mathrm{j}}+\mathrm{E}_{\mathrm{ij}}$; donde $\mathrm{Y}_{\mathrm{ij}}=$ respuesta del animal al recibo de la dieta; $\mathrm{U}=$ respuesta media general; $\mathrm{Si}=$ efecto del animal (aleatorio); $\mathrm{T}_{\mathrm{i}}=$ efecto de la dieta (fijo) y $\mathrm{E}_{\mathrm{ij}}=$ error (variación dentro de los animales).

Con los datos de PL, P, G y ST se verificaron los supuestos teóricos del análisis de varianza: 1) la prueba de Shapiro \& Wilk (1965), para la homogeneidad de varianza, 2) análisis de varianza y 3) comparación de medias con la prueba de Tukey $(\mathrm{p}=0,01)$. Los procedimientos y análisis estadísticos se realizaron con el programa SAS versión 9.4 (Statistical Analysis Systems Institute, 2016).

\section{Resultados}

Los suelos asociados a las localidades de evaluación de los genotipos de avena forrajera por ubicación y geomorfología se clasificaron: a) SRC (MOS, DUI, CER y CUC) como suelos ácidos del orden Inceptisoles y b) SRN (PAS, TAN, CUM y GUA) como suelos ácidos del orden Inceptisoles suborden Andeps, por su formación de origen volcánico con alto contenido de alófana.

En la SRC predominaron suelos con texturas franco arcillosa y franco arcilloso arenosa, ligeramente ácidos $(5,7)$, con contenido medio de materia orgánica $(5,1 \%$ a $7,7 \%)$ y un contenido alto de fósforo $(20,5$ a $38,9 \mathrm{mg}$ $\left.\mathrm{kg}^{-1}\right)$. Los suelos de la SRN mostraron una textura franco-arcillosa, un $\mathrm{pH}$ muy ácido $(5,2$ a 5,9$)$, con un contenido alto de fósforo $\left(17,15\right.$ a $\left.25,2 \mathrm{mg} \mathrm{kg}^{-1}\right)$ y alto de materia orgánica $(8,7 \%$ a $16,6 \%)$.

En relación con el calcio, magnesio y potasio en $\mathrm{cmol} \mathrm{kg}^{-1}$, el Ca presentó valores de alto a medio en los suelos de las dos subregiones con 4,3 a 15,2 para la SRC y de 3,3 a 8,8 para la SRN; Mg con valores de bajo a alto en la $\operatorname{SRC}(0,3$ a 3,6$)$ y de bajo a medio en la SRN $(0,6$ a 1,8$)$, y el $\mathrm{K}$ con valores de bajo a alto en ambas subregiones de 0,1 a 0,9 en la SRC y de 0,2 a 0,7 en la SRN (Cuadro 2).

\section{Prueba de evaluación agronómica: rendimiento de materia seca (RMS)}

El análisis de varianza combinado a través de localidades para rendimiento de materia seca (RMS), presentó diferencias altamente significativas $(\mathrm{p}=0,01)$ para localidad y genotipos y significativa $(\mathrm{p}=0,05)$ para la interacción genotipo x localidad. La mayor participación de la variación total del modelo estadístico para RMS se debió a los factores localidad y genotipo, con valores de $71,2 \%$ y $13,7 \%$, respectivamente, mientras que la menor participación se le atribuyó la interacción genotipo x localidad $(8,1 \%)$. Con base en el análisis AMMI, se estableció que los dos primeros componentes principales, CP1 Y CP2, con una significancia de $\alpha=0,01$, explicaron el 80,1 $\%$ de la interacción genotipo x localidad (Cuadro 3). Este resultado permite una interpretación confiable del comportamiento de la interacción genotipo x localidad, ya que un valor aceptable es una proporción mayor a 75 $\%$. El coeficiente de variación observado fue de $12,1 \%$, el cual se consideró aceptable. La mayor participación del factor localidad en la variación total del modelo estadístico para RMS a la variación de los promedios de RMS 
Cuadro 3. Análisis de varianza combinado para rendimiento de materia seca (RMS), de cinco genotipos de avena forrajera (Avena sativa L.) en ocho localidades en el Altiplano Cundiboyacense y de Nariño. Corporación Colombiana de Investigación Agropecuaria (AGROSAVIA). Colombia. 2016-2017.

Table 3. Combined analysis of variance for dry matter yield (DMY) of five forage oats (Avena sativa L.) genotypes in eight localities in the Altiplano Cundiboyacense and Nariño. Corporacion Colombiana de Investigacion Agropecuaria (AGROSAVIA). Colombia. 2016-2017.

\begin{tabular}{lccc}
\hline Fuente de variación & Grados de libertad & Rendimiento de materia seca & CP \% \\
\hline Repetición $(\mathbf{L})$ & 3 & 8,2 & \\
Localidad-L & 7 & $712,6 * *(71,2)$ & \\
Genotipo-G & 4 & $239,5 *(13,7)$ & \\
G x L & 28 & $20,0 *(8,1)$ & 54,1 \\
CP1 & 10 & $30,3 * *$ & 80,1 \\
CP2 & 8 & $17,2^{* *}$ & 97,6 \\
CP3 & 6 & 16,8 & 100,0 \\
CP4 & 4 & 4,6 & 100,0 \\
CP5 & 2 & 0,0 & \\
Error & 117 & 4,1 & \\
C. V \% & & 12,1 & \\
\hline
\end{tabular}

C.V: coeficiente de variación; *: diferencias significativas $(\mathrm{p}=0,05) * *$ : diferencias altamente significativas $(\mathrm{p}=0,01)$; valores entre paréntesis: porcentaje de participación del factor el modelo estadístico / C. V: coefficient of variation; *: significan differences ( $\mathrm{p}=0.05$ ); **: highly significant differences $(\mathrm{p}=0.01)$, values in parentheses: percentage of participation of the factor in the statisctical model.

se debió a un posible déficit de agua observado en dos de las ocho localidades del estudio (TAN y DUI) que no lograron satisfacer el $100 \%$ del requerimiento (Cuadro 2).

El gráfico biplot del AMMI que modeló la interacción genotipo x localidad con base en los dos primeros componentes principales, CP1 y CP2, con una participación de 54,1\% y 26,0 \%, respectivamente, permitió identificar genotipos con adaptación general o amplia a aquellos genotipos asociados con el punto cero y con adaptación específica a los genotipos asociados al vértice del vector de una o varias localidades, así como el aporte de cada localidad en la interacción genotipo x localidad (mayor longitud del vector mayor aporte en la interacción y viceversa) y la potencia del modelo para discriminar el comportamiento diferencial de los genotipos. Por su mayor y menor longitud del vector localidad, los ambientes MOS, DUI y CUM fueron los de mayor aporte a la interacción genotipo x localidad y PAS, CUC y TAN los de menor aporte (Figura 1).

El genotipo AV25-T con un rendimiento de materia seca de 20,9 t ha- ${ }^{-1}$, mostró una adaptación amplia o general a diferencia de los genotipos que presentaron adaptación específica AV25-S con 20,3 t ha-1 asociado con PAS y MOS, Avenar con 15,2 $\mathrm{t} \mathrm{ha}^{-1}$ asociado con GUA; Cajicá con 14,1 $\mathrm{t}^{-1}$ asociado con CUM, DUI y TAN y Cayuse con 14,6 t ha ${ }^{-1}$ asociado con CUM (Figura 1, Cuadro 4).

En relación con la potencia para reconocer el comportamiento diferencial del RMS con base en la mayor longitud del vector fueron MOS con 23,4 $\mathrm{tha}^{-1}$, CUM con 15,4 $\mathrm{t} \mathrm{ha}^{-1}$ y GUA con 12,9 $\mathrm{t} \mathrm{ha}^{-1} \mathrm{y}$ la menor potencia

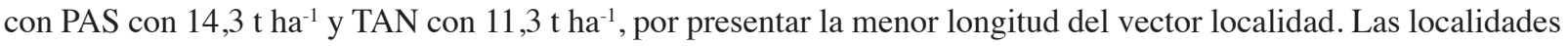
MOS y CER se constituyeron en los ambientes óptimos para la selección de genotipos con alto RMS (Cuadro 4, Figura 1).

La precipitación acumulada durante el periodo de siembra a cosecha de forraje verde, permitió agrupar las localidades en dos: a) localidades sin restricción de agua (SR) (MOS, CER, CUC, PAS, CUM, GUA), aquellas con $509,5 \mathrm{~mm}$ en promedio satisficieron en el $100 \%$ o cercano a este el requerimiento hídrico del cultivo de avena, y b) localidades con restricción de agua (CR) (DUI y TAN), las que con 255,3 mm solo lograron satisfacer la 


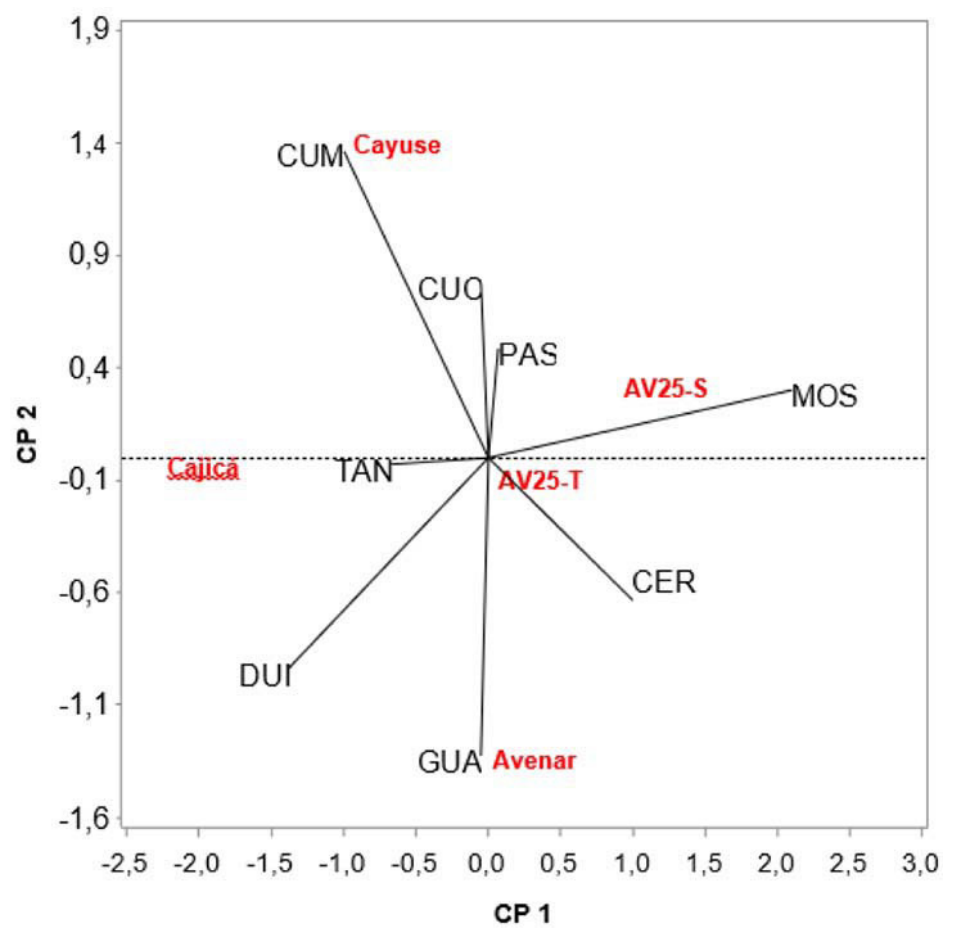

Figura 1. Representación de genotipos y localidades respecto a los dos primeros componentes, CP1 y CP2, del análisis AMMI para el rendimiento de materia seca de cinco genotipos de avena forrajera (Avena sativa L.) evaluados en ocho localidades de la zona andina colombiana. Corporación Colombiana de Investigación Agropecuaria (AGROSAVIA), 2016-2017.

MOS: Mosquera; CUC: Cucunubá; DUI: Duitama; CER: Cerinza; PAS: Pasto; TAN: Tangua; CUM: Cumbal; GUA: Guachucal.

Figure 1. Representation of genotypes and localities regarding the first two components, $\mathrm{CP} 1$ and $\mathrm{CP} 2$, of the AMMI analysis for the dry matter yield of five forage oats (Avena sativa) genotypes evaluated in eight environments of the Colombian Andean zone. Corporacion Colombiana de Investigacion Agropecuaria (AGROSAVIA), 2016-2017.

MOS: Mosquera; CUC: Cucunubá; DUI: Duitama; CER: Cerinza; PAS: Pasto; TAN: Tangua; CUM: Cumbal; GUA: Guachucal.

mitad del requerimiento hídrico (Cuadro 2 y 5). Este análisis comparativo permitió reconocer AV25-T y AV25-S con plasticidad fenotípica y posible tolerancia al déficit hídrico con una reducción de solo el 21,7 \% y 23,8 \%, respectivamente, a diferencia de la mayor reducción del RMS de casi el doble en los genotipos testigo Cayuse con $46,3 \%$, Avenar con 49,1\% y Cajicá con 58,3 \% (Cuadro 5).

\section{Prueba de evaluación agronómica: proteína, FDN y NTD}

El análisis de varianza combinado para proteína, FDN y NTD, presentó diferencias altamente significativas $(\mathrm{p}=0,01)$ para localidad y significativa $(\mathrm{p}=0,05)$ para genotipo e interacción genotipo $\mathrm{x}$ localidad. Los coeficientes de variación en todos los casos fueron menores a 10,0\%, para un nivel aceptable de confianza a los resultados obtenidos. No obstante, de haberse obtenido una interacción genotipo por localidad significativa, la participación del factor localidad fue tres veces mayor a la interacción genotipo por localidad para proteína $(56,8 \%$ vs $17,5 \%)$ y FDN $(50,1 \%$ vs 16,5$)$ y similar en NTD $(40,4 \%$ vs $39,1 \%)$ (Cuadro 6$)$.

La proteína presentó un intervalo de valores de 5,5\% a 10,0 \%, con una media para los genotipos de 8,2 \% para AV25-T, 8,1\% para AV25-S, iguales estadísticamente entre sí $(\mathrm{p}=0,05)$ y diferentes a los genotipos testigos comerciales Avenar con 7,4 \%, Cajicá con 7,3 y Cayuse con 7,2 \% (Cuadro 7). 
Cuadro 4. Rendimiento de materia seca $\left(\mathrm{t} \mathrm{ha}^{-1}\right)$ de cinco genotipos de avena (Avena sativa L.) forrajera en ocho localidades en el Altiplano Cundiboyacense y de Nariño. Corporación Colombiana de Investigación Agropecuaria (AGROSAVIA). Colombia. 20162017.

Table 4. Dry matter yield $\left(\mathrm{t} \mathrm{ha}^{-1}\right)$ of five forage oats (Avena sativa L.) genotypes in eight locations in the Altiplano Cundiboyacense and Nariño. Corporacion Colombiana de Investigacion Agropecuaria (AGROSAVIA). Colombia. 2016-2017.

\begin{tabular}{|c|c|c|c|c|c|c|c|c|c|}
\hline \multirow[b]{2}{*}{ Genotipo/subregión } & \multicolumn{4}{|c|}{ Cundiboyacense } & \multicolumn{4}{|c|}{ Nariño } & \multirow[t]{2}{*}{ Media } \\
\hline & MOS & DUI & CER & CUC & PAS & TAN & CUM & GUA & \\
\hline AV25-T & $27,8 \mathrm{a}$ & $18,2 \mathrm{a}$ & $27,9 \mathrm{a}$ & $26,3 \mathrm{a}$ & $16,8 \mathrm{a}$ & $16,4 \mathrm{a}$ & $17,4 \mathrm{a}$ & $16,5 \mathrm{a}$ & $20,9 \mathrm{a}$ \\
\hline AV25-S & $27,8 \mathrm{a}$ & $16,8 \mathrm{a}$ & $27,5 \mathrm{a}$ & $25,2 \mathrm{a}$ & $16,2 \mathrm{a}$ & $15,3 \mathrm{a}$ & $17,9 \mathrm{a}$ & $15,8 \mathrm{a}$ & $20,3 \mathrm{a}$ \\
\hline Avenar & $19,3 \mathrm{~b}$ & $9,3 \mathrm{~b}$ & $24,8 \mathrm{~b}$ & $20,7 \mathrm{~b}$ & $13,6 \mathrm{~b}$ & $8,3 \mathrm{~b}$ & $14,2 \mathrm{~b}$ & $11,2 \mathrm{~b}$ & $15,2 \mathrm{~b}$ \\
\hline Cajicá & $20,3 \mathrm{c}$ & $6,2 \mathrm{c}$ & $20,5 \mathrm{~b}$ & $20,4 \mathrm{c}$ & $14,9 \mathrm{~b}$ & $7,8 \mathrm{~b}$ & $12,8 \mathrm{~b}$ & $11,9 \mathrm{~b}$ & $14,1 \mathrm{~b}$ \\
\hline Cayuse & $22,1 \mathrm{~b}$ & $8,8 \mathrm{~b}$ & $20,5 \mathrm{~b}$ & $20,6 \mathrm{a}$ & $12,1 \mathrm{~b}$ & $8,9 \mathrm{~b}$ & $14,8 \mathrm{~b}$ & $9,2 \mathrm{c}$ & $14,6 \mathrm{~b}$ \\
\hline Media $^{1}$ & $23,4 \mathrm{a}$ & $11,9 \mathrm{c}$ & $24,2 \mathrm{a}$ & $22,6 \mathrm{a}$ & $14,7 \mathrm{~b}$ & $11,3 \mathrm{c}$ & $15,4 \mathrm{~b}$ & $12,9 \mathrm{c}$ & \\
\hline Media $^{2}$ & \multicolumn{4}{|c|}{$20,6 \mathrm{a}$} & \multicolumn{4}{|c|}{$13,4 \mathrm{~b}$} & \\
\hline C. V \% & 10,2 & 7,3 & 8,4 & 10,3 & 9,6 & 12,4 & 10,0 & 9,4 & \\
\hline
\end{tabular}

Media $^{1}$ : media localidades; media ${ }^{2}$ : media subregiones; C.V: coeficiente de variación; medías con letras iguales en las columnas no difieren estadísticamente ( $\mathrm{p} \leq 0,01$, Tukey); localidades: MOS: Mosquera; CUC: Cucunubá; DUI: Duitama; CER: Cerinza; PAS: Pasto; TAN: Tangua; CUM: Cumbal; GUA: Guachucal. / Mean ${ }^{1}$ : mean localities; mean ${ }^{2}$ : mean subregions; C.V: coefficient of variation; means with the same letters in the columns do not differ statistically ( $\leq \leq 0.01$, Tukey); Localities: MOS: Mosquera; CUC: Cucunuba; DUI: Duitama; CER: Cerinza; PAS: Pasto; TAN: Tangua; CUM: Cumbal; GUA: Guachucal.

Cuadro 5. Rendimiento relativo de materia seca $\left(\mathrm{t} \mathrm{ha}^{-1}\right)$ de cinco genotipos de avena forrajera (Avena sativa $\mathrm{L}$.) en dos condiciones de precipitación en el Altiplano Cundiboyacense y de Nariño. Corporación Colombiana de Investigación Agropecuaria (AGROSAVIA). Colombia. 2016-2017.

Table 5. Relative yield of dry matter $\left(\mathrm{t} \mathrm{ha}^{-1}\right)$ of five genotypes of forage oats (Avena sativa L.) genotypes in two precipitation conditions in the Altiplano Cundiboyacense and Nariño. Corporacion Colombiana de Investigacion Agropecuaria (AGROSAVIA). Colombia. $2016-2017$.

\begin{tabular}{|c|c|c|c|c|}
\hline \multirow{2}{*}{ Genotipo } & \multicolumn{2}{|c|}{ Localidades } & \multirow{2}{*}{$\begin{array}{c}\text { Reducción } \\
\text { t ha }^{-1}\end{array}$} & \multirow{2}{*}{$\begin{array}{c}\text { Reducción } \\
\%\end{array}$} \\
\hline & SR & $\mathbf{C R}$ & & \\
\hline AV25-T & 22,1 & 17,3 & 4,8 & 21,7 \\
\hline AV25-S & 21,7 & 16,0 & 5,7 & 23,8 \\
\hline Avenar & 17,3 & 8,8 & 8,5 & 49,1 \\
\hline Cajicá & 16,8 & 7,0 & 9,8 & 58,3 \\
\hline Cayuse & 16,6 & 8,9 & 7,7 & 46,3 \\
\hline Precipitación acumulada (mm) & 509,5 & 255,3 & & \\
\hline
\end{tabular}

SR: localidades sin restricción de agua (Mosquera, Cerinza, Cucunubá, Pasto, Cumbal, Guachucal); CR: localidades con restricción de agua (Duitama, Tangua). / SR: localities without water restriction (Mosquera, Cerinza, Cucunubá, Pasto, Cumbal, Guachucal); CR: localities with water restriction (Duitama, Tangua).

La mayor variación en proteína se observó entre localidades y subregiones. En la SRC con un intervalo de valores de 6,6\% a 9,9\% presentaron una media por localidad de 9,9\% en Mosquera, 9,7 \% en Cucunubá, $9,2 \%$ en Cerinza y $6,7 \%$ en Duitama, los tres primeros iguales estadísticamente entre sí $(\mathrm{p}=0,05)$ y diferentes estadísticamente $(\mathrm{p}=0,05)$ con Duitama. El segundo grupo en la SRN con un intervalo de valores de 5,3\% y 6,9\%, presentaron una media por localidad de 6,9\% en Cumbal, 6,8 \% en Guachucal, 6,4 \% en Pasto y 5,3\% en Tangua; 
Cuadro 6. Análisis de varianza combinado para proteína cruda (PC), fibra detergente neutro (FDN) y nutrientes totales digestibles (NTD) de cinco genotipos de avena forrajera (Avena sativa L.) en ocho localidades en el Altiplano Cundiboyacense y de Nariño. Corporación Colombiana de Investigación Agropecuaria (AGROSAVIA). Colombia. 2016-2017.

Table 6. Combined analysis of variance for crude protein (CP), neutral detergent fiber (NDF) and total digestible nutrients (TDN) of five forage oats (Avena sativa L.) genotypes in eight localities in the Altiplano Cundiboyacense and Nariño. Corporacion Colombiana de Investigacion Agropecuaria (AGROSAVIA). Colombia. 2016-2017.

\begin{tabular}{lcccc}
\hline & & \multicolumn{3}{c}{ Cuadrado medio (C.M) } \\
\cline { 3 - 5 } Fuente de variación & Grados de libertad & PC & FDN & NTD \\
\hline Repetición (L) & 3 & 16,2 & 40,2 & 42,6 \\
Localidad-L & 7 & $1250,3^{* *}(56,8)$ & $3418,2^{* *}(50,1)$ & $1420,5^{* *}(40,4)$ \\
Genotipo (G) & 4 & $710,7^{*}(18,4)$ & $1240,1^{*}(24,2)$ & $1084,3^{*}(18,5)$ \\
G x L & 28 & $96,3^{*}(17,5)$ & $120,4 *(16,5)$ & $327,6^{*}(39,1)$ \\
Error & 117 & 9,4 & 16,1 & 12,7 \\
C. V \% & & 9,2 & 5,3 & 4,8 \\
\hline
\end{tabular}

C.V: coeficiente de variación; *: diferencias significativas $(\mathrm{p}=0,05)$; **: diferencias altamente significativas $(\mathrm{p}=0,01)$; valores entre paréntesis porcentaje de participación del factor en el modelo. / C. V: coefficient of variation; *: significant differences ( $\mathrm{p}=0.05)$; **: highly significant differences $(\mathrm{p}=0.01)$; values in parentheses percentage of factor participation in the model.

los tres primeros iguales estadísticamente entre $s i ́(p=0,05)$ y diferentes estadísticamente $(\mathrm{p}=0,05)$ con Tangua. El valor medio por subregión fue de $8,9 \%$ para SRC y de 6,3\% para la SRN (Cuadro 7).

Cuadro 7. Proteína (\%) de cinco genotipos de avena forrajera (Avena sativa L.) en ocho localidades en el Altiplano Cundiboyacense y de Nariño. Corporación Colombiana de Investigación Agropecuaria (AGROSAVIA). Colombia. 2016-2017.

Table 7. Protein (\%) of five forage oats (Avena sativa L.) genotypes in eight localities in the Altiplano Cundiboyacense and Nariño. Corporacion Colombiana de Investigacion Agropecuaria (AGROSAVIA). Colombia. 2016-2017.

\begin{tabular}{|c|c|c|c|c|c|c|c|c|c|}
\hline \multirow{2}{*}{$\begin{array}{l}\text { Genotipo/ } \\
\text { Subregión }\end{array}$} & \multicolumn{4}{|c|}{ Cundiboyacense } & \multicolumn{4}{|c|}{ Nariño } & \multirow[b]{2}{*}{ Media } \\
\hline & MOS & DUI & CER & CUC & PAS & TAN & CUM & GUA & \\
\hline AV25-T & $10,6 \mathrm{a}$ & $7,4 \mathrm{a}$ & 9,9 a & $10,9 \mathrm{a}$ & $6,6 \mathrm{a}$ & $5,0 \mathrm{~b}$ & $7,7 \mathrm{~b}$ & $7,1 \mathrm{a}$ & $8,2 \mathrm{a}$ \\
\hline$A v-25-S$ & $10,9 \mathrm{a}$ & $7,2 \mathrm{a}$ & $9,8 \mathrm{a}$ & $10,0 \mathrm{a}$ & $6,4 \mathrm{a}$ & $5,1 \mathrm{~b}$ & $7,9 \mathrm{~b}$ & $7,4 \mathrm{a}$ & $8,1 \mathrm{a}$ \\
\hline Avenar & $9,2 \mathrm{a}$ & $6,3 \mathrm{a}$ & $9,3 \mathrm{a}$ & 9,5 a & $6,5 \mathrm{a}$ & $5,5 \mathrm{a}$ & 6,4 a & $6,8 \mathrm{a}$ & $7,4 \mathrm{~b}$ \\
\hline Cajicá & $9,4 \mathrm{a}$ & 6,3 a & $8,9 \mathrm{~b}$ & $9,0 \mathrm{a}$ & $6,4 \mathrm{a}$ & $5,4 \mathrm{~b}$ & $6,2 \mathrm{a}$ & $6,6 \mathrm{a}$ & $7,3 \mathrm{~b}$ \\
\hline Cayuse & 9,6 a & $6,1 \mathrm{a}$ & $8,1 \mathrm{a}$ & $9,2 \mathrm{a}$ & $6,3 \mathrm{a}$ & $5,3 \mathrm{a}$ & $6,3 \mathrm{a}$ & 6,3 a & $7,2 \mathrm{~b}$ \\
\hline Media1 & $9,9 \mathrm{a}$ & $6,7 \mathrm{~b}$ & $9,2 \mathrm{a}$ & $9,7 \mathrm{a}$ & $6,4 \mathrm{~b}$ & $5,3 \mathrm{c}$ & $6,9 \mathrm{~b}$ & $6,8 \mathrm{~b}$ & \\
\hline Media2 & \multicolumn{4}{|c|}{8,9 a } & \multicolumn{4}{|c|}{$6,3 \mathrm{~b}$} & \\
\hline C.V \% & 4,5 & 4,8 & 4,2 & 3,9 & 4,4 & 4,2 & 4,1 & 4,0 & \\
\hline
\end{tabular}

Media $^{1}$ : media localidades; media ${ }^{2}$ : media subregiones; C.V: coeficiente de variación; medias con letras iguales en las columnas no difieren estadísticamente ( $\leq \leq 0,01$, Tukey); Localidades: MOS: Mosquera; CUC: Cucunubá; DUI: Duitama; CER: cerinza; PAS: pasto; TAN: Tangua; CUM: Cumbal; GUA: Guachucal. / Mean ${ }^{1}$ : mean localities; mean ${ }^{2}$ : mean subregions; C.V: coefficient of variation; means with the same letters in the columns do not differ statistically ( $\mathrm{p} \leq 0.01$, Tukey); Localities: MOS:Mosquera; CUC: Cucunubá; DUI: Duitama; CER: Cerinza; PAS: Pasto; TAN: Tangua; CUM: Cumbal; GUA: Guachucal. 
La fibra en detergente neutro (FDN) presentó un intervalo de valores de 50,1\% a 65,5\% con una media para los genotipos de 56,6 \% para Cajicá, 56,2 \% para Cayuse, 55,8 \% para AV25-T, 55,3\% para AV25-S iguales estadísticamente entre sí $(\mathrm{p}=0,05)$ y diferentes estadísticamente $(\mathrm{p}=0,05)$ a Avenar con 53,3\% (Cuadro 8).

Cuadro 8. Fibra en detergente neutro (\%) de cinco genotipos de avena forrajera (Avena sativa L.) en ocho localidades en el Altiplano Cundiboyacense y de Nariño. Corporación Colombiana de Investigación Agropecuaria (AGROSAVIA). Colombia. $2016-2017$.

Table 8. Neutral detergent fiber (\%) of five genotypes of forage oats (Avena sativa L.) genotypes in eight localities in the Altiplano Cundiboyacense and Nariño. Corporacion Colombiana de Investigacion Agropecuaria (AGROSAVIA). Colombia. $2016-2017$.

\begin{tabular}{|c|c|c|c|c|c|c|c|c|c|}
\hline \multirow{2}{*}{$\begin{array}{l}\text { Genotipo/ } \\
\text { Subregión }\end{array}$} & \multicolumn{4}{|c|}{ Cundiboyacense } & \multicolumn{4}{|c|}{ Nariño } & \multirow[b]{2}{*}{ Media } \\
\hline & MOS & DUI & CER & CUC & PAS & TAN & CUM & GUA & \\
\hline AV25-T & $59,6 \mathrm{~b}$ & $54,9 \mathrm{a}$ & $58,3 \mathrm{~b}$ & $60,2 \mathrm{~b}$ & $52,9 \mathrm{a}$ & $51,3 \mathrm{a}$ & $54,7 \mathrm{a}$ & $54,2 \mathrm{a}$ & $55,8 \mathrm{a}$ \\
\hline Av25-S & $59,1 \mathrm{~b}$ & $54,0 \mathrm{a}$ & $58,5 \mathrm{~b}$ & $60,4 \mathrm{~b}$ & $52,4 \mathrm{a}$ & $50,1 \mathrm{a}$ & $54,8 \mathrm{a}$ & $53,3 \mathrm{a}$ & $55,3 \mathrm{a}$ \\
\hline Avenar & $56,9 \mathrm{~b}$ & $52,4 \mathrm{~b}$ & $51,5 \mathrm{c}$ & $60,9 \mathrm{~b}$ & $51,7 \mathrm{a}$ & $50,1 \mathrm{a}$ & $51,1 \mathrm{a}$ & $52,2 \mathrm{a}$ & $53,4 \mathrm{~b}$ \\
\hline Cajicá & $62,7 \mathrm{a}$ & $56,7 \mathrm{a}$ & $65,3 \mathrm{a}$ & $60,0 \mathrm{~b}$ & $52,5 \mathrm{a}$ & $50,5 \mathrm{a}$ & $52,7 \mathrm{a}$ & $52,1 \mathrm{a}$ & $56,6 \mathrm{a}$ \\
\hline Cayuse & $59,7 \mathrm{~b}$ & $52,9 \mathrm{~b}$ & $65,5 \mathrm{a}$ & $62,9 \mathrm{a}$ & $52,1 \mathrm{a}$ & $50,9 \mathrm{a}$ & $54,3 \mathrm{a}$ & $50,9 \mathrm{~b}$ & $56,2 \mathrm{a}$ \\
\hline Media $^{1}$ & 59,6 a & $54,2 \mathrm{~b}$ & 59,8 a & $60,9 \mathrm{a}$ & $52,3 \mathrm{a}$ & 50,6 a & $53,5 \mathrm{~b}$ & $52,5 \mathrm{~b}$ & \\
\hline Media $^{2}$ & \multicolumn{4}{|c|}{$58,6 \mathrm{a}$} & \multicolumn{4}{|c|}{$52,2 \mathrm{~b}$} & \\
\hline C.V \% & 8,4 & 8,0 & 6,8 & 8,0 & 8,9 & 9,2 & 9,2 & 8,7 & \\
\hline
\end{tabular}

Media $^{1}$ : media localidades; media ${ }^{2}$ : media subregiones; C.V: coeficiente de variación; medias con letras iguales en las columnas no difieren estadísticamente (P $\leq 0,01$, Tukey); Localidades: MOS: Mosquera; CUC: Cucunubá; DUI: Duitama; CER: Cerinza; PAS: Pasto; TAN: Tangua; CUM: Cumbal; GUA: Guachucal / Mean ${ }^{1}$ : mean localities; mean²: mean subregions C.V: coefficient of variation; means with the same letters in the columns do not differ statistically ( $\mathrm{P} \leq 0.01$, Tukey); Localities: MOS: Mosquera; CUC: Cucunubá; DUI: Duitama; CER: Cerinza; PAS: Pasto; TAN: Tangua; CUM: Cumbal; GUA: Guachucal.

La mayor variación de fibra en detergente neutro (FDN) se observó entre localidades y subregiones. En la SRC con un intervalo de valores de 54,2\% a 60,9\%, presentaron una media por localidad de 60,9\% en Cucunubá, 59,8 \% en Cerinza, 59,6\% en Mosquera y 54,2 \% en Duitama, los tres primeros iguales estadísticamente entre sí $(\mathrm{p}=0,05)$ y diferentes estadísticamente $(\mathrm{p}=0,05)$ con Duitama. El segundo grupo en la SRN, con un intervalo de valores de 50,6 \% a 53,5\%, presentaron una media por localidad de 53,5\% en Cumbal, 52,5\% en Guachucal, $52,3 \%$ en Pasto y 50,6 \% en Tangua, los tres primeros iguales estadísticamente entre sí $(\mathrm{p}=0,05)$ y diferentes estadísticamente $(\mathrm{p}=0,05)$ con Tangua. El valor medio por subregión fue de 58,6 \% para SRC y de 52,2 \% para la SRN (Cuadro 8).

El total de nutrientes digestibles (NTD) presentó un intervalo de valores de 50,9\% a 64,9\%, con una media para los genotipos de 57,8 \% para AV25-T, 53,9\% para Cayuse, 53,9\% para AV25-S, 53,1\% para Avenar, iguales estadísticamente entre sí $(\mathrm{p}=0,05)$ y diferentes estadísticamente $(\mathrm{p}=0,05)$ a Cajicá con 55,0\% (Cuadro 9). La variación de FDN no fue alta entre localidades y subregiones. En la SRC, con un intervalo de valores de 55,8\% a 56,3\%, presentaron una media por localidad de 56,3\% en Cerinza, 55,8 \% en Cucunubá, 55,0 \% en Mosquera y $52,5 \%$ en Duitama los tres primeros iguales estadísticamente entre sí $(\mathrm{p}=0,05)$ y diferentes estadísticamente $(\mathrm{p}=0,05)$ con Duitama. El segundo grupo en la SRN con un intervalo de valores de $51,1 \%$ a $54,8 \%$, presentaron una media por localidad de 54,8 \% en Guachucal, 53,7 \% en Pasto, 52,9\% en Cumbal y 51,1\% en Tangua, los tres primeros iguales estadísticamente entre sí $(\mathrm{p}=0,05)$ y diferentes estadísticamente $(\mathrm{p}=0,05)$ con Tangua. El valor medio por subregión fue de 54,9\% para SRC y de 53,1\% para la SRN, iguales estadísticamente entre sí $(\mathrm{p}=0,05)$ (Cuadro 9). 
Cuadro 9. Nutrientes totales digestibles (\%) de cinco genotipos de avena forrajera (Avena sativa L.) en ocho localidades en el Altiplano Cundiboyacense y de Nariño. Corporación Colombiana de Investigación Agropecuaria (AGROSAVIA). Colombia. $2016-2017$.

Table 9. Total digestible nutrients (\%) of five forage oats (Avena sativa L.) genotypes in eight localities in the Altiplano Cundiboyacense and Nariño. Corporacion Colombiana de Investigacion Agropecuaria (AGROSAVIA). Colombia. 2016-2017.

\begin{tabular}{|c|c|c|c|c|c|c|c|c|c|}
\hline \multirow{2}{*}{$\begin{array}{l}\text { Genotipo/ } \\
\text { subregión }\end{array}$} & \multicolumn{4}{|c|}{ Cundiboyacense } & \multicolumn{4}{|c|}{ Nariño } & \multirow[b]{2}{*}{ Media } \\
\hline & MOS & DUI & CER & CUC & PAS & TAN & CUM & GUA & \\
\hline AV25-T & $64,9 \mathrm{a}$ & $54,3 \mathrm{a}$ & $63,9 \mathrm{a}$ & $58,9 \mathrm{a}$ & $56,8 \mathrm{a}$ & $51,9 \mathrm{a}$ & $54,9 \mathrm{a}$ & $56,6 \mathrm{a}$ & $57,8 \mathrm{a}$ \\
\hline Av25-S & $54,2 \mathrm{~b}$ & $52,2 \mathrm{a}$ & $54,2 \mathrm{~b}$ & $58,1 \mathrm{a}$ & $53,3 \mathrm{~b}$ & $51,5 \mathrm{a}$ & $52,7 \mathrm{a}$ & $55,5 \mathrm{a}$ & $53,9 \mathrm{~b}$ \\
\hline Avenar & $53,9 \mathrm{~b}$ & $51,2 \mathrm{a}$ & $53,4 \mathrm{~b}$ & $56,4 \mathrm{a}$ & $52,2 \mathrm{~b}$ & $50,9 \mathrm{a}$ & $52,1 \mathrm{a}$ & $54,6 \mathrm{a}$ & $53,1 \mathrm{~b}$ \\
\hline Cajicá & $53,6 \mathrm{~b}$ & $52,1 \mathrm{a}$ & $55,7 \mathrm{~b}$ & $51,2 \mathrm{~b}$ & $52,9 \mathrm{~b}$ & $51,7 \mathrm{a}$ & $53,4 \mathrm{a}$ & $54,0 \mathrm{a}$ & $53,0 \mathrm{~b}$ \\
\hline Cayuse & $53,8 \mathrm{~b}$ & $52,6 \mathrm{a}$ & $54,4 \mathrm{~b}$ & $58,2 \mathrm{a}$ & $53,1 \mathrm{a}$ & $51,3 \mathrm{a}$ & $52,6 \mathrm{a}$ & $55,3 \mathrm{a}$ & $53,9 \mathrm{~b}$ \\
\hline Media $^{1}$ & $55,0 \mathrm{a}$ & $52,5 \mathrm{~b}$ & 56,3 a & $55,8 \mathrm{a}$ & $53,7 \mathrm{a}$ & $51,1 \mathrm{~b}$ & 52,9 a & $54,8 \mathrm{a}$ & \\
\hline Media $^{2}$ & \multicolumn{4}{|c|}{$54,9 \mathrm{a}$} & \multicolumn{5}{|c|}{$53,1 \mathrm{a}$} \\
\hline C.V \% & 4,8 & 6,0 & 6,2 & 7,0 & 4,4 & 4,0 & 8,6 & 6,0 & \\
\hline
\end{tabular}

Media $^{1}$ : media localidades; media²: media subregiones; C.V: coeficiente de variación; medias con letras iguales en las columnas no difieren estadísticamente ( $\mathrm{p} \leq 0,01$, Tukey); Localidades: MOS: Mosquera; CUC: Cucunubá; DUI: Duitama; CER: Cerinza; PAS: Pasto; TAN: Tangua; CUM: Cumbal; GUA: Guachucal. / Mean ${ }^{1}$ : mean localities; mean²: mean subregions, C.V: coefficient of variation; means with the same letters in the columns do not differ statistically ( $\leq 0.01$, Tukey); Localities: MOS: Mosquera; CUC: Cucunubá; DUI: Duitama; CER: Cerinza; PAS: Pasto; TAN: Tangua; CUM: Cumbal; GUA: Guachucal.

\section{Análisis comparativo por subregiones: Cundiboyacense y de Nariño}

El rendimiento de materia seca (RMS), la proteína, la fibra en detergente neutro (FDN) y el total de nutrientes digestibles (NTD) presentaron valores diferenciados en las localidades asociadas con la SRC y la SRN. En la primera subregión el RMS de 18,3 t ha ${ }^{-1}$ fue superior y diferente estadísticamente $(\mathrm{p}=0,05)$ al obtenido en la segunda subregión con 12,6 t ha ${ }^{-1}$. Similar situación se observó en la SRC para proteína, FDN y NTD con valores de $8,2 \%, 56,4 \%$ y $54,3 \%$, respectivamente, superiores estadísticamente $(\mathrm{p}=0,05)$ al obtenido en la SRN con 6,0 $\%, 52,4 \%$ y $51,8 \%$, respetivamente (Cuadro 10 ).

Los resultados diferenciados de RMS, proteína, FDN y NTD, podrían estar asociados con las diferencias en la altitud, temperatura y precipitación y que no tuvieron control en la experimentación; a diferencia del factor suelo que sí tuvo control en la experimentación, ya que fueron uniformizados con el encalamiento y la fertilización edáfica.

\section{Respuesta animal}

Para el factor dieta las variables producción de leche y grasa presentaron diferencias significativas $(\mathrm{p}=0,05)$, mientras que proteína y sólidos totales no mostraron diferencias estadísticas $(\mathrm{p}=0,05)$. Todos los casos los coeficientes de variación fueron menores al 15,0\%, lo que representó buen grado de confiabilidad de los datos. La dieta solo con kikuyo (D1) presentó un rendimiento de leche de 22,7 $1 \mathrm{vaca}^{-1}$ día $^{-1}$, menor y diferente estadísticamente $(\mathrm{p}=0,05)$ al rendimiento obtenido con las dietas que incluyeron ensilaje de AV25-T $35 \%$ (D2) y 65 $\%$ (D3), con 29,7 1 vaca $^{-1}$ día $^{-1}$ y 27,21 vaca $^{-1}$ día $^{-1}$, respectivamente, estas dos últimas dietas iguales estadísticamente entre sí $(\mathrm{p}=0,05)$. El rendimiento de leche con $35 \%$ y $65 \%$ de suplemento de ensilaje de AV25-T, representó un incremento de 7,0 y 4,5 L/vaca/día, respectivamente (Cuadro 11). 
Cuadro 10. Factores de productividad, calidad de ensilaje de avena forrajera (Avena sativa L.) relacionados con el clima y suelo en dos subregiones del trópico alto colombiano. Corporación Colombiana de Investigación Agropecuaria (AGROSAVIA). Colombia. 2016-2017.

Table 10. Productivity factors, quality of forage oat (Avena sativa L.) silage related to climate and soil in two subregions of the Colombian high tropics. Corporacion Colombiana de Investigacion Agropecuaria (AGROSAVIA). Colombia. 2016-2017.

\begin{tabular}{lcc}
\hline Productividad y calidad & SRC & SRN \\
\hline Rendimiento materia seca $\left(\mathrm{t} \mathrm{ha}^{-1}\right)$ & $18,3 \mathrm{a}$ & $12,6 \mathrm{~b}$ \\
Proteína $(\%)$ & $8,2 \mathrm{a}$ & $6,0 \mathrm{~b}$ \\
Fibra detergente neutro (\%) & $56,4 \mathrm{a}$ & $52,4 \mathrm{~b}$ \\
Nutrientes totales digestibles $(\%)$ & $54,3 \mathrm{a}$ & $51,8 \mathrm{~b}$ \\
Clima & & \\
Altitud (msnm) & 2677 & 3018 \\
Temperatura $\left({ }^{\circ} \mathrm{C}\right)$ & 17,2 & 13,7 \\
Precipitación acumulada $(\mathrm{mm})$ & 458 & 342 \\
Suelo & & \\
pH & 5,7 & 5,4 \\
Materia orgánica $(\%)$ & 6,3 & 11,2 \\
Fósforo $\left(\mathrm{mg} \mathrm{kg}^{-1}\right)$ & 27,0 & 19,5 \\
\hline
\end{tabular}

SRC: subregión Cundiboyacense; SRN: subregión Nariño; medias con letras iguales en las columnas no difieren estadísticamente ( $\mathrm{p} \leq 0,01$, Tukey). / SRC: Cundiboyacense subregion; SRN: Nariño subregion; means with the same letters in the columns do not differ statistically $(\mathrm{p} \leq 0.01$, Tukey).

Cuadro 11. Producción de leche, proteína, grasa, sólidos totales, nitrógeno ureico y cambio de peso animal por efecto de tres dietas en vacas Holstein (Bos primigenius Taurus). Corporación Colombiana de Investigación Agropecuaria (AGROSAVIA). Tibasosa, Altiplano Cundiboyasense, Colombia. 2017-2018.

Table 11. Milk production, protein, fat, total solids, urea nitrogen, and change in animal weight due to the effect of three diets in Holstein cows (Bos primigenius Taurus). Corporacion Colombiana de Investigacion Agropecuaria (AGROSAVIA). Tibasosa, Altiplano Cundiboyasense, Colombia. 2017-2018.

\begin{tabular}{lccccc}
\hline Dieta & $\begin{array}{c}\text { Producción leche } \\
\text { L/vaca/día }\end{array}$ & $\begin{array}{c}\text { Proteína } \\
\mathbf{\%}\end{array}$ & $\begin{array}{c}\text { Grasa } \\
\mathbf{\%}\end{array}$ & $\begin{array}{c}\text { Sólidos totales } \\
\mathbf{\%}\end{array}$ & $\begin{array}{c}\text { Cambio peso } \\
\text { animal (kg) }\end{array}$ \\
\hline D1 & $22,7 \mathrm{~b}$ & $3,0 \mathrm{a}$ & $3,1 \mathrm{~b}$ & $11,7 \mathrm{a}$ & $-0,50 \mathrm{c}$ \\
D2 & $29,7 \mathrm{a}$ & $2,9 \mathrm{a}$ & $3,9 \mathrm{a}$ & $11,6 \mathrm{a}$ & $0,10 \mathrm{~b}$ \\
D3 & $27,2 \mathrm{a}$ & $2,7 \mathrm{a}$ & $3,2 \mathrm{a}$ & $11,1 \mathrm{a}$ & $0,25 \mathrm{a}$ \\
& & & & \\
Vaca & $70,5 \mathrm{~ns}$ & $7,2 \mathrm{~ns}$ & $6,8 \mathrm{~ns}$ & $4,4 \mathrm{~ns}$ & $0,10 \mathrm{~ns}$ \\
Dieta & $76,9 *$ & $18,6 \mathrm{~ns}$ & $14,2 *$ & $10,5 \mathrm{~ns}$ & $0,46 *$ \\
Error & 14,1 & 2,6 & 3,2 & 1,8 & 0,07 \\
C.V $\%$ & 3,8 & 9,6 & 5,8 & 4,9 & 4,6 \\
\hline
\end{tabular}

D1: testigo solo pradera; D2: ensilage de AV25-T (35\%); D3: ensilage de AV25-T (65 \%); *: significativo al $5 \%$ de probabilidad y ns: no significativo; medias con letras iguales en las columnas no difieren estadísticamente ( $\mathrm{x} \leq 0,01$, Tukey). / D1: only grassland control; D2: AV25-T silage (35\%); D3: AV25-T silage (65\%); *: significant at $5 \%$ of probability and ns: not significant; means with the same letters in the columns do not differ statistically ( $\mathrm{p} \leq 0.01$, Tukey). 
La proteína y los sólidos totales en leche no presentaron diferencias estadísticas $(\mathrm{p}=0,05)$ para el factor dieta. La proteína obtenida solo con kikuyo fue de 3,0 \% y con suplmentación de $35 \%$ y $65 \%$ de ensilaje de AV25-T fueron de $2,9 \%$ y 2,7 \%, respectivamente. Similar comportamiento se observó en los sólidos totales, con valores iguales estadísticamente $(\mathrm{p}=0,05)$ de $11,7 \%, 11,6 \%$ y $11,1 \%$ con kikuyo, $35 \%$ y $65 \%$ de AV25-T, respectivamente (Cuadro 11).

El contenido de grasa en leche presentó el mayor valor de 3,9\% con la inclusión de $35 \%$ de ensilaje de AV25-T en la dieta y diferente estadísticamente $(\mathrm{p}=0,05)$ con el valor de $3,1 \%$ solo kikuyo y $3,2 \%$ con $65 \%$ de ensilaje. Se observó un incremento significativo de $0,8 \%$ con el cambio de la dieta de kikuyo por $35 \%$ de ensilaje de AV25-T y se redujo en $0,7 \%$ al pasar de $3,9 \%$ a $3,2 \%$ con $35 \%$ a $65 \%$ de AV25-T, respectivamente. El efecto de la dieta en el cambio de peso animal fue estadísticamente significativo $(\mathrm{p}=0,05)$. De hecho, el peso animal con kikuyo presentó una reducción significativa de $-0,50 \mathrm{~kg}$, a diferencia de la ganancia de peso obtenida con $35 \%$ y $65 \%$ de ensilaje de AV25-T con 0,10 kg y 0,25 kg, respectivamente (Cuadro 11).

\section{Discusión}

El rendimiento en materia seca (RMS) y los componentes de calidad composicional de la proteína, la fibra en detergente neutro (FDN) y los nutrientes totales digestibles (NTD) en condiciones de ensilaje, presentaron en el modelo estadístico una mayor participación del factor localidad en relación con los factores genotipo e interacción genotipo x localidad. No obstante, de haberse presentado una interacción significativa para la interacción G x L, no se descartó el análisis del efecto localidad. Sobresalió las diferencias significativas en RMS, proteína, FDN y no significativa para NTD entre las subregiones SRC y SRN.

En la prueba de evaluación agronómica (PEA) las localidades y subregiones el clima y suelo fueron heterogéneas; no obstante, fueron más notorias las diferencias de clima en la altitud, temperatura y precipitación, factores que tienen una interacción compleja e inciden en el crecimiento y desarrollo y en los procesos de absorción, transporte de agua y nutrientes en la avena (Canales et al., 2019; Jinqiu et al., 2021; Rispail et al., 2018). De hecho, la altitud fue menor en la SRC (2066 msnm) y mayor en la SRN (3018 msnm), la temperatura fue mayor en la SRC con $17,2{ }^{\circ} \mathrm{C}$ y menor en la $\mathrm{SRN}$ con $13,7^{\circ} \mathrm{C}$ y similar situación para la precipitación en SRC con 458 mm mayor a la ocurrida en la SRN con $342 \mathrm{~mm}$.

Existen estudios relacionados, aunque pocos en avena, entre la altitud y la eco-fisiología de la planta y producción de biomasa. En esta investigación, en la SCN con mayor altitud el RMS presentó una reducción de 5,7 t ha ${ }^{-1}$ en comparación con la obtenida en la subregión con menor altitud (SRN) $\left(18,3 \mathrm{t} \mathrm{ha}^{-1} \mathrm{vs} 12,6 \mathrm{t} \mathrm{ha}^{-1}\right)$. Este resultado podría estar en concordancia con los expresado por Jinqiu et al. (2021), quienes obtuvieron modificaciones estructurales y fisiológicas en avena por efecto de la altitud (2918 msnm vs $2080 \mathrm{msnm}$ ); altitudes mayores presentaron mayores incrementos en la actividad enzimática antioxidante y de oxígeno reactivo, menor apertura estomática, menor fotosíntesis y su consecuente disminución en el rendimiento.

La precipitación no sujeta de control en esta investigación, fue la que ocasionó quizás la mayor heterogeneidad entre localidades y subregiones, sustentado en la mayor participación de la variación del factor localidad en la variación total del modelo estadístico con 71,2 \% 56,8 \%, 50,1 \% y 43,1 \% para RMS, proteína, FDN y NTD, respectivamente. Con base en el agua requerida de $400 \mathrm{~mm}$ para una producción de 60,0 a 80,0 t ha ${ }^{-1}$ de forraje verde en avena (Hussain et al., 2010; Terán-Chaves et al., 2014), esta se satisfizo en seis de las ocho localidades en un $100 \%$ o muy cercano al $100 \%$ y solo en dos, Duitama y Tangua, lo fue en un $75 \%$ y $53 \%$, respectivamente.

La condición sin restricción (SR) en las localidades presentó quizás mayor disponibilidad de agua y mejor nutrición del cultivo de avena asociado con mayor acumulación de biomasa y formación de compuestos para calidad bromatológica, relación observada y reportada en diferentes cultivos donde se incluyó la avena (Wang 
et al., 2020; Zhang et al., 2019). La comparación del RMS y su reducción por efecto de condición diferencial de humedad de suelo (sin restricción-SR y con restricción CR de agua) evidenció la presencia de plasticidad fenotípica en AV25-T y AV25-S con una reducción del 23,5 \% y 28,1 \%, respectivamente, menor en un $50 \%$ a las observadas en Avenar, Cajicá y Cayuse con 53,4 \%, 53,6 \% y 53,5\%, respectivamente.

Existe y está reportada la asociación entre la plasticidad fenotípica con la resistencia a la roya de la hoja (Puccinia coronata f. sp avenae) (Montilla-Bascón et al., 2013). El mayor rendimiento de forraje verde se asoció con la menor severidad y reacción varietal de resistencia de AV25-T y AV25-S en la PEA (SRC y SRN), lo que se observó en los altiplanos Cundiboyacense y de Nariño y se reportó por Campuzano et al. (2020), quienes confirmaron esta relación.

La plasticidad fenotípica está reportada en varias especies [trigo (Triticum vulgare), sorgo (Sorghum bicolor L.), cebada (Hordeum vulgare L.) y arroz (Oryza sativa L.)] (Martínez-Sánchez, 2018), incluida la avena (Avena sativa L.) (Akçura \& Çeri, 2011; Canales et al., 2019; Rispail et al., 2018; Sadras et al., 2017; Sánchez-Martín et al., 2015; 2017). Los genotipos AV25-T y AV25-S mostraron mayor RMS y mejor calidad bromatológica del ensilaje (proteína, FDN y NTD). El RMS en $\mathrm{t} \mathrm{ha}^{-1}$ en ambos genotipos (AV25-T de 21,3 y AV25-S de 20,7) se consideraron altos y superiores a los reportados para avena forrajera en Etiopia de 2,0 a 2,2 (Jabessa et al., 2020), en México de 5,4 (Mendoza-Pedroza et al., 2021), en la India 6,1 a 9,1 (Singh et al., 2018; Tulu et al., 2020), en Pakistán de 7,7 a 15,6 (Ali et al., 2016; Ayub et al., 2011), y menores a lo reportado en Pakistán por Bilal et al. (2017) de 25,8 y por Mwendia et al. (2017) en Kenia con 22,0.

Los resultados obtenidos en proteína con el uso de ensilajes de AV25-T de 8,2 \% y AV25-S de 8,1 \%), fueron superiores al reporte en Perú de 7,9\% (Mamani Paredes \& Cotacallapa Gutiérrez, 2018), 9,9 en Pakistán (Ayub et al., 2011), similar a la india con $8,1 \%$ (Kapoor \& Singh, 2020) y menores en relación con otro estudio en la India con 10,4 a 13,1 \% (Singh et al., 2018) y en la SRN (Colombia) de 9,3\% (Castro-Rincón, 2020) y de 7,9\% en la SRC (Colombia) (López et al., 2012). Los valores de FDN (AV25-T de 55,8 \% y AV25-S de 55,3 \%) fueron superiores a los presentados en Nariño (Colombia) de 49,0 \% (Castro-Rincón et al., 2020) y de 48,0 \% (CardonaIglesias et al., 2019), en México de 43,9 \% a 49,1 \% (Ramírez-Ordoñez et al., 2013) y en Perú de 46,9 \% (Mamani Paredes \& Cotacallapa Gutiérrez, 2018); similares a lo reportado en la SRC de 54,8 \% (López et al., 2012) y menores a lo reportado por Kapoor \& Singh (2020) de 65,4 \%. En relación con NTD (AV25-T de 53,9 \% y AV25-S $53,9 \%$ ) los datos obtenidos fueron menores a los reportado por Casto-Rincón (2020) en la SRN (Colombia) con $71,0 \%$ y en la SRC (Colombia) de 68,7 \% (López et al., 2012).

En esta investigación se muestran los efectos de la inclusión de la dieta de ensilaje de AV25-T y la sustitución del pasto kikuyo en la alimentación animal durante la época de verano en la SRC con un cambio significativo en la producción de leche, grasa, así como un incremento en el peso del animal. Solo con el pasto kikuyo el rendimiento de leche fue menor que con el uso de ensilaje, ya que a medida que esta dieta fue sustituida con el ensilaje de AV25-T se obtuvieron 7,0 L/vaca/día más con $35 \%$ de ensilaje y 4,5 L/vaca/día más con $65 \%$.

Los resultados de este estudio concuerdan con lo encontrado en Vietnam en vacas Holstein, con un incremento de $16,3 \mathrm{~kg}$ /día a 17,3 kg/día, cuando se reemplazó el pasto tropical en pastoreo por ensilaje de avena (Salgado et al., 2013); en Costa Rica, en vacas Jersey con el reemplazo del pasto kikuyo con ensilaje de avena forrajera, el rendimiento de leche se incrementó de 14,8 a 15,6 L/vaca/día (Yuste et al., 2016), en condiciones similares en la SRN (Colombia) el reemplazo del pastoreo de kikuyo con $35 \%$ de ensilaje de avena representó 4,0\% más en la producción de leche (Castro-Rincón et al., 2020) y en vacas Kiwi x Holstein aumentó de 9,4 L/vaca/día a 10,0 L/ vaca/día (Cardona-Iglesias et al., 2019).

Las variables de proteína y sólidos totales, pese a no haber presentado diferencias entre las dietas, sí se observó una tendencia de disminución de $0,1 \%$ y $0,3 \%$ al reemplazar el kikuyo con $35 \%$ y $65 \%$ con ensilaje de AV25-T, mientras que los sólidos totales mostraron una reducción de $0,1 \%$ y 0,6 \%. Sumado a esto, la concentración de grasa se incrementó en $0,8 \%$ cuando se sustituyó el kikuyo con $35 \%$ de ensilaje de AV25-T y se redujo en 0,7 
\% cuando se pasó de $35 \%$ a $65 \%$ de ensilaje de AV25-T. La mejor calidad bromatológica de ensilaje AV25-T en proteína $(8,2 \%)$ y NTD $(53,8)$, contribuyó a una mejor relación proteico-energético y mayor digestibilidad. Estos resultados concuerdan de forma parcial con los reportados por otros investigadores, por ejemplo, Mojica et al. (2009) encontraron una reducción en la proteína del 0,2 \% y un aumento de 0,15\% cuando se sustituyó el kikuyo con 0,7 \% y 1,4\% de ensilaje de avena. López et al. (2012) reportaron, en condiciones similares en la SRC, incrementos en la proteína, la grasa y los sólidos totales en la medida que se sustituyó el kikuyo con el ensilaje de avena forrajera.

En este estudio, las dietas de $35 \%$ y $65 \%$ de ensilaje de AV25-T, permitieron un incremento significativo de $0,10 \mathrm{~kg}$ y $0,25 \mathrm{~kg}$, en relación con la reducción de $-0,5 \mathrm{~kg}$ con la dieta solo kikuyo. Este resultado concuerda con los encontrados por López et al. (2012), donde la suplementación con ensilaje de avena en reemplazo del pastoreo con kikuyo, permitió un incremento en el peso animal de $540 \mathrm{~kg}$ a $558 \mathrm{~kg}$ y está sustentado en la correlación positiva entre la ganancia de peso animal con la proteína-energía, donde los ensilajes aventajaron en forma significativa al kikuyo que presentó bajos contenidos de materia seca y energía (Correa et al., 2008).

\section{Conclusiones}

El único genotipo de avena que presentó estabilidad fenotípica amplia y mostró el mayor RMS y proteína y menor FDN, en relación con los tres testigos comerciales Avenar, Cajicá y Cayuse fue AV25-T.

Con la inclusión del ensilaje de avena forrajera de AV25-T como estrategia alimenticia en época de verano, se obtuvo el mayor rendimiento de leche de $29,7 \mathrm{~L} / \mathrm{vaca} /$ día, lo que representó un incremento en 7,0 L/vaca/día en relación con el testigo kikuyo y 2,5 L/vaca/día más de leche en relación con la inclusión de 65 \% de ensilaje; en ambos casos sin detrimento de la calidad bromatológica de la lecha en su proteína y sólidos totales.

El contenido de grasa en leche dependió de la concentración de la dieta; con el $35 \%$ de ensilaje de AV25-T se obtuvo $0,8 \%$ más en relación con la dieta con kikuyo y $0,7 \%$ más que con el $65 \%$ de ensilaje de AV25-S.

El uso de la avena forrajera AV25-T en ensilaje en el trópico alto colombiano, permitió en vacas lecheras Holstein mitigar los efectos negativos de la época de sequía, con un aumento en el peso animal y mayor producción de leche y grasa.

\section{Agradecimientos}

La información contenida en este artículo formó parte del informe técnico titulado "Evaluación agronómica de dos genotipos de avena forrajera (AV25-T y AV25-S) para la región Andina, subregión natural del Altiplano Cundiboyacense y altiplano de Nariño, 2016-2017”. Esta investigación fue posible por el apoyo económico de la Cuenta Nacional de Carne y Leche, el Ministerio de Agricultura y Desarrollo Rural de Colombia (MADR) y la Corporación Colombiana de Investigación Agropecuaria (AGROSAVIA).

\section{Referencias}

Akçura, M., \& Çeri, S. (2011). Evaluation of drought tolerance indices for selection on Turkish oat (Avena sativa L.) landraces under various environmental conditions. Žemdirbystè, 98(2), 157-166. 
Ali, M., Mahmood, S., Ibrahem., \& Mizel, M. S. (2016). Determined the oat (Avena sativa L.) genotype sresponding to different planting dates in southern Iraq. International Journal of Agronomy and Agricultural Research, 9(6), 33-43.

Amézquita, M. C. (1986). Consideraciones sobre planeación, diseño y análisis de experimentos de pastoreo. En C. Lascano, \& E. Pizarro (Eds.), Evaluación de pasturas con animales. Alternativas metodológicas (pp. 13-42). Centro Internacional de Agricultura Tropical. http://hdl.handle.net/20.500.12324/29851

Ariza-Nieto, C., Mayorga, O., Mojica, B., Parra, D., \& Afanador-Tellez, G. (2017). Use of LOCAL algorithm with near infrared spectroscopy in forage resources for grazing systems in Colombia. Journal of Near Infrared Spectroscopia, 26(1), 44-52. https://doi.org/10.1177/0967033517746900

Arteaga, L. E., \& Burbano, J. E. (2017). Efectos del cambio climático: Una mirada al Campo. Revista de Ciencias Agrícolas, 35(2), 79-91. https://doi.org/10.22267/rcia.183502.93

Ayub, M., Shehzad, M., Nadeem, M. A., Pervez, M., Naeem, H., \& Sarwar, N. (2011). Comparative study of forage oan quality of different oat (Avena sativa L.) varieties under agroecological conditions of Faisalabad, Pakistán. African Journal of Agricultural Research, 6(14), 3388-3391.

Bilal, M., Ayuh, M Tariq, M., \& Nadeem, M. A. (2017). Dry matter yield and forage quality traits of oat (Avena sativa L.) under integrative use of microbial and synthetic source of nitrogen. Journal of the Saudi Society of Agricultural Sciences, 16(3), 236-241. https://doi.org/10.1016/j.jssas.2015.08.002

Campuzano, L. F., Castro, E., Castillo, J., Torres, D., Nieto, D., \& Portillo, P. (2020). Altoandina: nueva variedad de avena forrajera para la zona Andina en Colombia. Agronomía Mesoamericana, 31(3), 581-595. https://doi.org/10.15517/ am.v31i3.38999

Canales, F. J., Montilla-Bascón, G., Rispail, N., \& Prats, E. (2019) Salicylic acid regulates polyamine biosynthesis during drought responses in oat. Plant Signaling \& Behavior, 14(10), Article e1651183. https://doi.org/10.1080/15592324.2 019.1651183

Cardona-Iglesias, J. J., Castro-Rincón, E., Valenzuela-Chiran, M., Hernández-Oviedo, F., \& Avellaneda-Avellaneda, Y. (2019). Efecto del ensílaje de avena sobre la productividad de vacas lactantes en Nariño, Colombia. Revista Científica, FVCLUZ, 29(3), 165-169.

Carulla, J. E., \& Ortega, E. (2016). Dairy production systems of Colombia: challenges and opportunities. Archivos Latinoamericanos de Producción Animal, 24(2), 83-87.

Castro-Rincón, E., Cardona-Iglesias, J. I., Hernández-Oviedo, F., \& Valenzuela, M. (2020). Efecto del ensílaje de Avena sativa L. en la productividad de vacas lactantes en pastoreo. Pastos y Forrajes, 43(2), 150-158.

Correa, H. J., Pabón, M. L., \& Carulla, J. E. (2008). Valor nutricional del pasto kikuyo (Pennisetum clandestinum Hoechst Ex Chiov.) para la producción de leche en Colombia (Una revisión): I - Composición química y digestibilidad ruminal y posruminal. Livestock Research for Rural Development, 20, Article 59. http://www.lrrd.org/lrrd20/4/corra20059.htm

Departamento de Administración Nacional de Estadística. (2015). Encuesta nacional agropecuaria. https://www. dane.gov.co/ files/investigaciones/agropecuario/enda/ena/2015/presentacion_ena_2015.pdf

Guerrero-Riascos, R. (Ed.). (1998). Fertilización de cultivos en clima frío. Monómeros Colombos Venezolanos. Recuperado abril 2021, de http://www.monomeros.com/descargas/dpmanualfrio.pdf

Agron. Mesoam. 33(1): Artículo 44508, 2022

ISSN 2215-3608 doi:10.15517/am.v33i1.44508 
Gutiérrez, H. J. 1991. Clasificaciones climáticas. Instituto Colombiano de Hidrología Meteorología y Adecuación de Tierras. http://documentacion.ideam.gov.co/cgi-bin/koha/opac-detail.pl?biblionumber=2267\&shelfbrowse_itemnumber=2459

Hussain, A., Khan, S., Bakhsh, A., Imran, M., \& Ansar, M. (2010). Variability in fodder production potential of exotic oats (Avena sativa) genotypes under irrigated conditions. Journal of Agriculture Research, 48(1), 65-71.

Instituto de Hidrología, Meteorología y Estudios Ambientales. (2017). Clasificaciones climáticas Colombia. http://www. ideam.gov.co/documents/21021/21789/climas+\%5BModo+de+compatibilidad\%5D.pdf/d8c85704-a07a-4290-ba65f2042ce99ff 9

Instituto Colombiano Agropecuario. (2020). Resolución No. 067516: Por medio de la cual se establecen los requisitos para la inscripción de los cultivares en el Registro Nacional de Cultivares Comerciales y se dictan otras disposiciones. https:// www.ica.gov.co/getattachment/6b7dbbd1-ff9b-4eea-a936-fe57f421ea98/2020R67516.aspx

Jabessa, T., Amare,Z., \& Dejene, G. (2020). Adaptability study of oat (Avena sativa) genotypes for their agronomic performance and nutritional value in the midland area of eastern Quji, Adola, Southern Oromia, Ethiopia. Asian Journal of Agriculture and Allied Sciences, 3(1), 9-12. https://www.ikppress.org/index.php/AJAAS/article/view/4977

Jinqiu, Y., Bing, L., Tingting, S., Jinglei H., Zelai, K., Lu, L., Wenhua H., Tao, H., Xinyu, H., Zengqing, L., Guowen, C, \& Yajun, C. (2021). Integrated physiological transcriptomic, analysis responses to altitude stress in oat. Fontiers in Genetics, 12, Article 638683. https://doi.org/10.21203/rs.3.rs-37220/v1

Kapoor, R., \& Singh, T. P. (2020). OL 12: A New High Yielding Fodder Oat Variety Released for Punjab State, India. International Journal of Current Microbiology and Applied Sciences, 9(4), 3174-3178. https://doi.org/10.20546/ ijcmas.2020.904.369

López, D., Saavedra, G. F., Arreaza, L. C., Muñoz, S. G., \& Rodríguez, C. E. (2012). Evaluación de sistemas de alimentación como estrategia para afrontar la estacionalidad en Ganado lechero. Ciencia y Agricultura, 9(2), 39-46.

Lui, K. J. (2016). Crossover designs: testing, estimation, and sample size. John Wiley and Sons.

Mamani Paredes, J., \& Cotacallapa Gutiérrez, F. H. 2018. Rendimiento y calidad nutricional de avena forrajera en la región de Puno. Revista de Investigación Altoandina, 20,384-400. https://doi.org/10.18271/ria.2018.415

Martínez-Sánchez, J., Espinosa-Paz, N., Ramírez-Córdova A. L., Camas-Gómez, R., \& Villegas-Aparicio, Y. (2018). Expresión fenotípica y estabilidad en poblaciones de maíz nativo de Chiapas. Revista Mexicana de Agroecosistemas, 5(1), 1-11.

Mendoza-Pedroza, S. I., Sánchez-Espinoza, J., Álvarez-Vázquez, P., Sosa-Montes, E., Maldonado-Peralta, M. Á., \& GarayMartínez, J. R. (2021). Yield estimation of forage oat (Avena sativa L.) Chihuahua variety: ruler and plate methods. Agro Productividad, 14(1), 75-80. https://doi.org/10.32854/agrop.v14i1.1939

Mojica, J. E., Castro, E., Leon, J. M., Cardenas, E. A., Pabon, M. L., \& Carulla, J. E. (2009). Efecto de la oferta de pasto kikuyo y ensilaje de avena sobre la producción y calidad composicional de la leche bovina. Ciencia y Tecnología Agropecuaria, 10(1),81-90. https://doi.org/10.21930/rcta.vol10_num1_art:132

Montilla-Bascón, G., Sánchez-Martín, J., Rispail, N., Rubiales, D., Mur, L., Langdon, T., Griffiths, I., Howarth C., \& Prats, E. (2013). Genetic diversity and population structure among oat cultivars and landraces. Plant Molecular Biology Reporter, 31, 1305-1314. https://doi.org/10.1007/s11105-013-0598-8

Mwendia, S, W., Maass, B. L., Njenga, D. G., Nyakundi, F. N., \& Notenbaert, A. M. O. (2017). Evaluating oat cultivars for dairy forage production in the central Kenyan highlands. African Journal of Range \& Forage Science, 34(3), $145-155$. 
Ramírez-Ordóñes, S., Domínguez-Díaz, D., Salmerón-Zamora, J. J., Villalobos-Villalobos, G., \& Ortega-Gutiérrez, J. A. (2013). Producción y calidad del forraje de variedades de avena en función del sistema de siembra y de la etapa de madurez al corte. Revista Fitotecnia Mexicana, 36(4), 395-403.

Rispail, N., Montilla-Bascon, G., Sanchez-Martin, J., Flores, F., Howarth, C., Langdon, T., Rubiales, D., \& Prats, E. (2018). Multi-environmental trial reveal genetics plasticity of oat agronomic traits associated with climate variable changes. Frontier in Plant Science, 9, Article 1358. https://doi.org.10.3389/fpls.2018.01358

Sadras, V., Mahadevan, M., \& Zwer, P. (2017). Oat phenotypes for drought adaptation and yield potential. Field Crops Research, 212, 135-144. https://doi.org/10.1016/j.fcr.2017.07.014

Sánchez-Martín, J., Heald, J., Kingston-Smith, A., Winters, A., Rubiales, D., Sanz, M., Mur, L. A., \& Prats E. A. (2015). A metabolomic study in oats (Avena sativa) highlights a drought tolerance mechanism based upon salicylate signaling pathways and the modulation of carbon, antioxidant and photo-oxidative metabolism. Plant Cell Environmental, 38(7), 1434-1452. https://doi.org/10.1111/pce.12501

Sánchez-Martín, J., Rispail, N., Flores, F., \& Emeran, A. (2017). Higher rust resistance and similar yield of oat landraces versus cultivars under high temperature and drought. Agronomy for Sustainable Development, 37, Article 3. https://doi. org/10.1007/s13593-016-0407-5

Salgado, P., Thang, V. Q., Thu, T. V., Trach, N. X., Cuong V. C., Lecomte, P., \& Richard, D. (2013). Oats (Avena strigosa) as winter forage for dairy cows in Vietnam: an on-farm study. Tropical Animal Health and Production, 45(2), 561-568. https://doi.org/10.1007/s11250-012-0260-8

Shapiro, S. S., \& Wilk, M. B. (1965). An analysis of variance test for normality (complete samples). Biometrika, 52(3-4), 591-611. https://doi.org/10.1093/biomet/52.3-4.591

Sharma, P., Tiwari, S., Tripathi, N., \& Mehta, A. K. (2016). Polymorphism analysis in advanced mutant population of oat (Avena sativa L.) using ISSR markers. Physiology and Molecular Biology of Plants, 22, 115-120. https://doi.org/10.1007/ s12298-015-0333-Z

Singh, D., Chauhan, A., \& Chaudhary, A. (2018). Relative performance of oat forage varieties for seed production, economics, and fodder yield under central Gujarat conditions. Forage Research, 44(3), 181-191.

Statistical Analysis Systems Institute. (2016). The SAS system for Windows (Release 9.4.). SAS Institute Inc.

Terán-Chaves, C., Murcia Contreras, G., \& García-Prats, A. (2014). Determinación de requerimientos hídricos para avena forrajera (Avena sativa L.) y raigrás (Lolium perenne) en la Sabana de Bogotá (Colombia). Revista Facultad Nacional de Agronomía, 67(2), 1157-1159.

Tulu, A., Diribsa, M., \& Temesgen, W. (2020). Evaluation of seven oat (Avena sativa) genotypes for biomass yield and quality parameters under different locations of Western Oromia, Ethiopia. Advances in Agricultura, 2020, Article 822344. https://doi.org/10.1155/2020/8822344

Vargas Martínez, J. de J., Sierra Alarcón, A. M., Mancipe Muñoz, E. A., \& Avellaneda Avellaneda, Y. (2018). El kikuyo, una gramínea presente en los sistemas de rumiantes en trópico alto colombiano. Revista CES Medicina Veterinaria Zootecnia, 13(2), 137-156. https://doi.org/10.21615/cesmvz.13.2.4

Wada, A., Shawle, K., \& Gemiyo, D. (2019). Biomass yield and nutritional quality of different oat varieties (Avena sativa) grown under irrigation condition in Sodo Zuriya Distric, Wolaita Zone, Ethiopia. Agricultural Research, 20(4), 1-10. https://doi.org/10.19080/ARTOAS.2019.20556138

Agron. Mesoam. 33(1): Artículo 44508, 2022

ISSN 2215-3608 doi:10.15517/am.v33i1.44508 
Wang, Z., Jiang, H., \& Shen, Y. (2020). Forage production and soil water balance in oat and common vetch sole crops and intercrops cultivated in the summer-autumn fallow season on the Chinese Loess Plateau. European Journal Agronomy, 115, Article 126042. https://doi.org/10.1016/j.eja.2020.126042

Yuste, S.; Sánchez, W.; Vega, A., \& Guada, J. A. (2016). Effects of substitution of kikuyu forage by oat silage on milk production and quality in dairy cows. Unión de Entidades Españolas de Ciencia Animal. https://ueeca.chil.me/ attachment/55c0b531-ff80-4c83-89e6-793001e264af

Zadoks, J. C., Chang, T. T., \& Konzak, C. F. (1974). A decimal code for the growth stages of cereals. Weed Research, 14, 415-421. https://doi.org/10.1111/j.1365-3180.1974.tb01084.x

Zhang, A., Christensen, M., Nari, Z., Whish, J. P. M., Bell, L. W., Wang, J., Wang, Z., \& Sim, R. (2019). Plant development and solar radiation interception on four annual forage plants in response to sowing date in semiarid environment. Industrial Crops and Products, 131, 41-53. https://doi.org/10.1016/j.indcrop.2019.01.028 